NISTIR 8017

\title{
A Measurement Metric for Forensic Latent Fingerprint Preprocessing
}

Haiying Guan

Andrew Dienstfrey

Mary Theofanos

Brian Stanton 
NISTIR 8017

\title{
A Measurement Metric for Forensic Latent Fingerprint Preprocessing
}

\author{
Haiying Guan \\ Brian Stanton \\ Information Access Division \\ Information Technology Laboratory \\ Andrew Dienstfrey \\ Applied and Computational Mathematics Division \\ Information Technology Laboratory \\ Mary Theofanos \\ Office of Data and Informatics \\ Material Measurement Laboratory
}

This publication is available free of charge from:

http://dx.doi.org/10.6028/NIST.IR.8017

July 2014

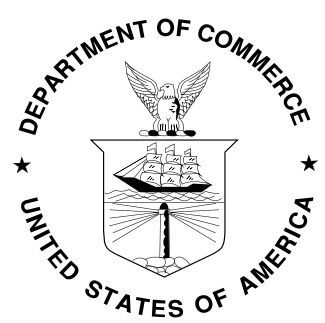

U.S. Department of Commerce Penny Pritzker, Secretary

National Institute of Standards and Technology Willie E. May, Acting Under Secretary of Commerce for Standards and Technology and Acting Director 


\section{TABLE OF CONTENTS}

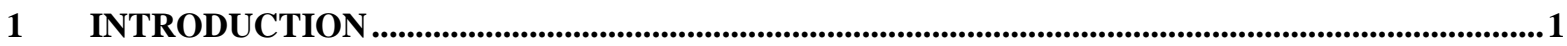

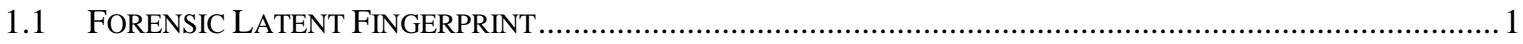

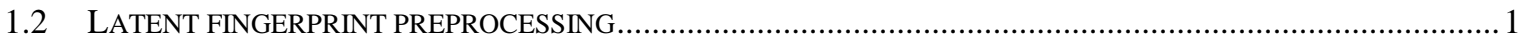

1.3 THE SOFTWARE AND TOOLS FOR LATENT FINGERPRINT PREPROCESSING..............................................

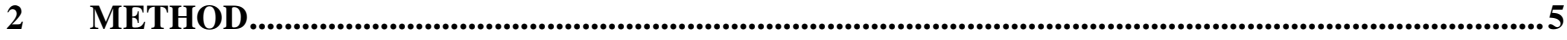

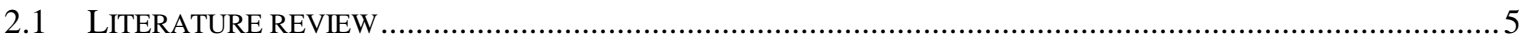

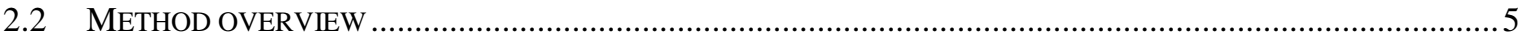

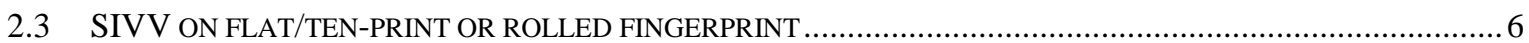

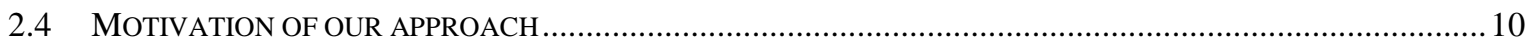

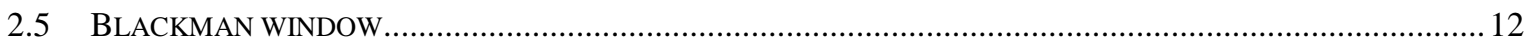

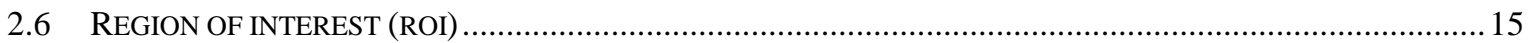

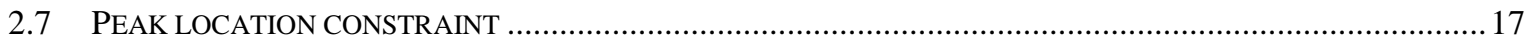

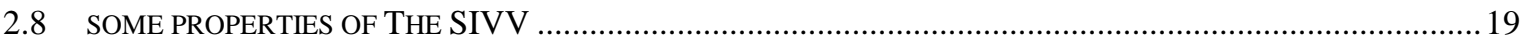

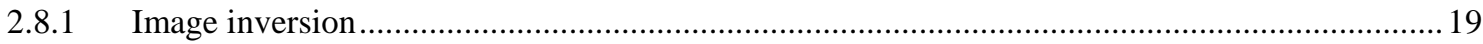



$3 \quad$ EXPERIMENTAL RESULTS ..............................................................................................................24

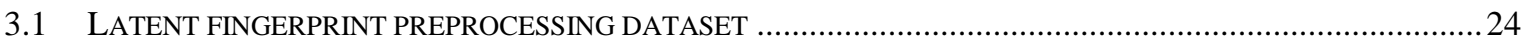

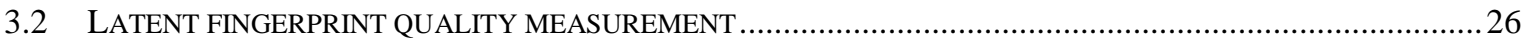

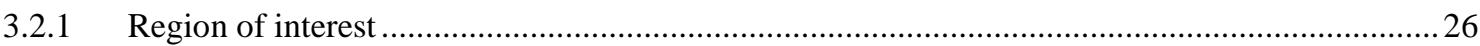

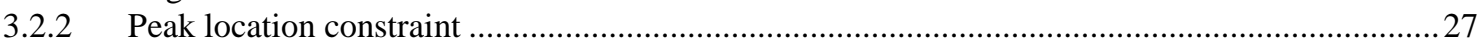

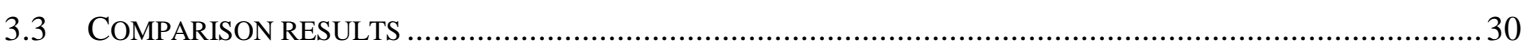

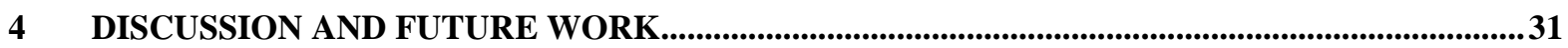

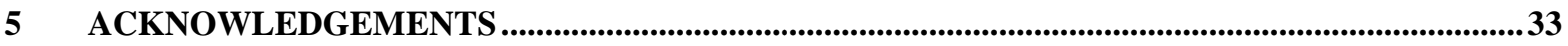

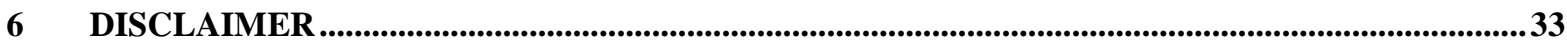

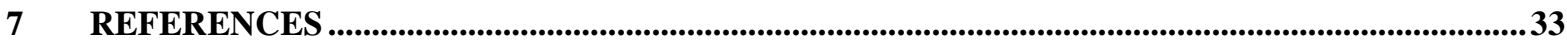

APPENDIX A: THE SIVV PEAK ON SYNTHETIC IMAGES ......................................................................37 


\section{LIST OF TABLES AND FIGURES}

TABLE 1: THE COMPARISON OF DIFFERENT ALGORITHMS WITH DIFFERENT OPTIONS

TABLE 2: SIVV PEAK LOCATIONS ON THE IMAGES WITH THE STRIPES PATTERN (IMAGE SIZE 512×512 PIXELS) ... 40

TABLE 3: SIVV PEAK LOCATIONS ON THE LARGE IMAGES WITH THE STRIPES PATTERN (IMAGE SIZE 1024×1024 PIXELS)

TABLE 4: THE RELATIONSHIP BETWEEN PEAK LOCATIONS AND PPI.

TABLE 5: THE PEAK LOCATIONS GIVEN THE STRIPE PIXEL DISTANCE.

FIGURE 1: AN EXAMPLE OF FORENSIC LATENT FINGERPRINT PREPROCESSING. .2

FIGURE 2: THE NECESSITY OF LATENT FINGERPRINT PREPROCESSING. .3

FIGURE 3: DISTINCT ENDPOINTS OF FORENSIC LATENT FINGERPRINT PREPROCESSING .4

FIGURE 4: SIVV ALGORITHM. 8

FIGURE 5: AN EXAMPLE OF SIVV FEATURE ON A ROLLED FINGERPRINT IMAGE. . .9

FIGURE 6: AN EXAMPLE OF THE SIVV FEATURE ON A LATENT FINGERPRINT IMAGE. 10

FIGURE 7: EXAMPLES OF THE SIVV FEATURE ON LATENT FINGERPRINT IMAGES. 12

FigURE 8: BLACKMAN Window ( $\mathrm{A}=0.16)$. 13

FIGURE 9: THE COMPARISON OF SIVV CURVES WITH DIFFERENT INPUT OPTIONS. 14

FIGURE 10: THE SELECTION OF REGION OF INTEREST. 15

FIGURE 11: AN EXAMPLE OF REGION OF INTEREST 16

FIGURE 12: AN EXAMPLE OF SIVV PEAK LOCATION CONSTRAINT. 18

FIGURE 13: THE ORIGINAL IMAGE AND ITS INVERT IMAGE OF WHITE POWER DEVELOPED PRINTS. 20

FIGURE 14: THE COMPARISON OF SIVV CURVES OF AN IMAGE AND ITS INVERT IMAGE. .21

FIGURE 15: THE IMAGE WITH DIFFERENT LEVELS OF IID RANDOM NOISE. 22

FIGURE 16: THE IMAGE MATRICES WITH RANDOM NOISE. 23

FIGURE 17: THE COMPARISON OF SIVV CURVES OF AN IMAGE WITH DIFFERENT LEVELS OF IID RANDOM NOISE. 24 FIGURE 18: THE LATENT FINGERPRINT PREPROCESSING IMAGE PAIRS FROM A TRAINING COURSE (SIX TYPES). ...25 
FIGURE 19: THE PROPOSED ALGORITHM FOR LATENT FINGERPRINT QUALITY MEASUREMENT..............................26

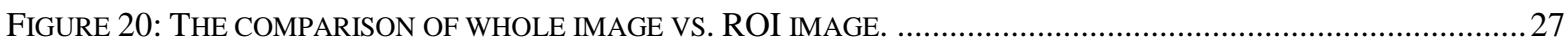

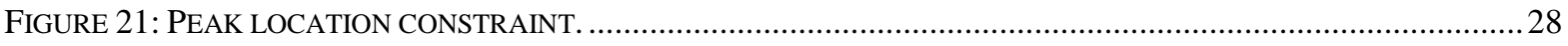

FIGURE 22: PROPOSED PROCEDURE FOR LATENT FINGERPRINT SIVV FEATURE DETECTION ................................ 29

FIGURE 23: THE FREQUENCY FEATURE OF LATENT FINGERPRINT USING THE PROPOSED ALGORITHM. .................... 30

FIGURE 24: QUANTITATIVE COMPARISON OF LATENT FINGERPRINT IMAGE QUALITY. ......................................... 32

FIGURE 25: SIVV ON THE IMAGES WITH THE STRIPES PATTERN (IMAGE SIZE 512×512 PIXELS).......................... 39

FIGURE 26: SIVV ON THE LARGE IMAGES WITH THE STRIPES PATTERN (IMAGE SIZE 1024×1024 PIXELS)..............40

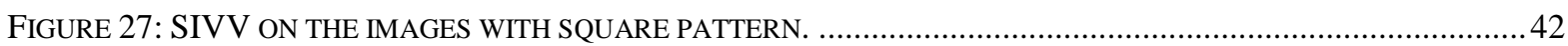




\section{EXECUTIVE SUMMARY}

Although fingerprint mark-up and identification are well-studied fields, forensic fingerprint image preprocessing is still a relatively new domain in need of further scientific study and development of best practice guidance. Latent fingerprint image preprocessing is a common step in the forensic analysis workflow that is performed to improve image quality for subsequent identification analysis while simultaneously ensuring data integrity. Due to the low quality of the latent fingerprint images, preprocessing is especially crucial to the success of the final fingerprint identification in the forensic fingerprint image examination. In this report, we isolate the forensic fingerprint image preprocessing step for more detailed analysis.

First we provide a brief review of latent fingerprint image preprocessing. We then turn to the problem of defining an image-based quality metric suitable for analysis of forensic latent fingerprint preprocessing. More precisely, we propose to extend Spectral Image Validation and Verification (SIVV) [1] to serve as a metric for latent fingerprint image quality measurement. SIVV analysis was originally developed to differentiate ten-print or rolled fingerprint images from other non-fingerprint images such as face or iris images. Several modifications are required to extend SIVV analysis to the latent space. We implement and test this new SIVV-based metric for latent fingerprint image quality and use it to measure the performance of the forensic latent fingerprint preprocessing step. Preliminary results show that the new metric can provide positive indications of both latent fingerprint image quality and the performance of the fingerprint preprocessing.

\section{Keywords:}

Forensic latent fingerprint image, Latent fingerprint image quality measurement, Spectral Image Validation and Verification Metric (SIVV), Latent fingerprint preprocessing, Fingerprint image enhancement. 


\section{INTRODUCTION}

\subsection{FORENSIC LATENT FINGERPRINT}

Fingerprints have been used to identify persons for centuries. As one of the most prevalent and powerful types of forensic evidence that can be recovered during the investigation of a crime, fingerprints have been routinely used for person identification in crime scenes.

There are different types of fingerprints. Ten-print and rolled fingerprints are captured on a fingerprint card or by special electronic devices. These fingerprint capture devices generally have built-in monitoring systems to guarantee the image quality. By contrast to this controlled capture process, the term latent fingerprints, or latents, refer to fingerprint impressions that are left unintentionally. Generally latents are partial prints lifted from surfaces of objects found at crime scenes that are touched or grasped by a person's fingers. Lifting of latent fingerprints involves complicated processes. Several chemical and physical development techniques are available to enhance the visibility of the friction ridge detail including: photographing the prints under different light sources, dusting with powders, and chemical processing. Ideally, the enhanced print can be lifted from the substrate and transferred to a secondary backing material that serves to improve the contrast of the ridges with respect to the background. While these development techniques improve fingerprint features, generally latents are of significantly poorer quality compared to rolled prints. This can depend on the substrate of the original latent impression. For example, when attempting to lift prints from porous paper substrates such as newsprint, magazines, and wallpaper, the lifted fingerprints' quality may be very low in some cases, and they are not usable for recognition even after preprocessing. Moreover, latent fingerprint backgrounds can exhibit diverse combinations of color, design, and texture that can mask the identity and spatial configuration of minutiae in a questioned print. In spite of these drawbacks, latent fingerprints are extremely useful in forensics to investigate crime scenes [22][23][24][25]. As a widely used biometric, latent fingerprints support an irreplaceable functionality: fingerprint recognition. Fingerprint recognition is a technique that can link latents to suspects whose fingerprints were previously enrolled in ten-print or rolled fingerprints databases, or to link to latent fingerprints from different crime scenes.

\subsection{LATENT FINGERPRINT PREPROCESSING}

The performance of a fingerprint recognition system is heavily dependent on the quality of the collected fingerprint images. This poses a problem for latent fingerprints as their image quality is generally low due to the combination of difficulties in lifting the print from substrate and image contamination by complex background noise. As a result, fingerprint structure such as minutiae and ridges may not be clearly visible to the human eye of a fingerprint examiner, nor easily detected by the algorithms in automatic matching systems. 
Due to the poor quality of latent fingerprint images, digital image preprocessing is a necessary step in the forensic analysis workflow [2]. Image preprocessing is performed to increase latent fingerprint image quality. Some of the common transformations employed in service of this goal include: color management, contrast adjustment, edge enhancement, background suppression, and noise filtration [3] [4] [5] [6] [7] [8]. Figure 1 shows an example of forensic latent fingerprint image preprocessing: the color image on the left is the image directly collected from the crime scene, and the gray-level image on the right is the image after preprocessing.
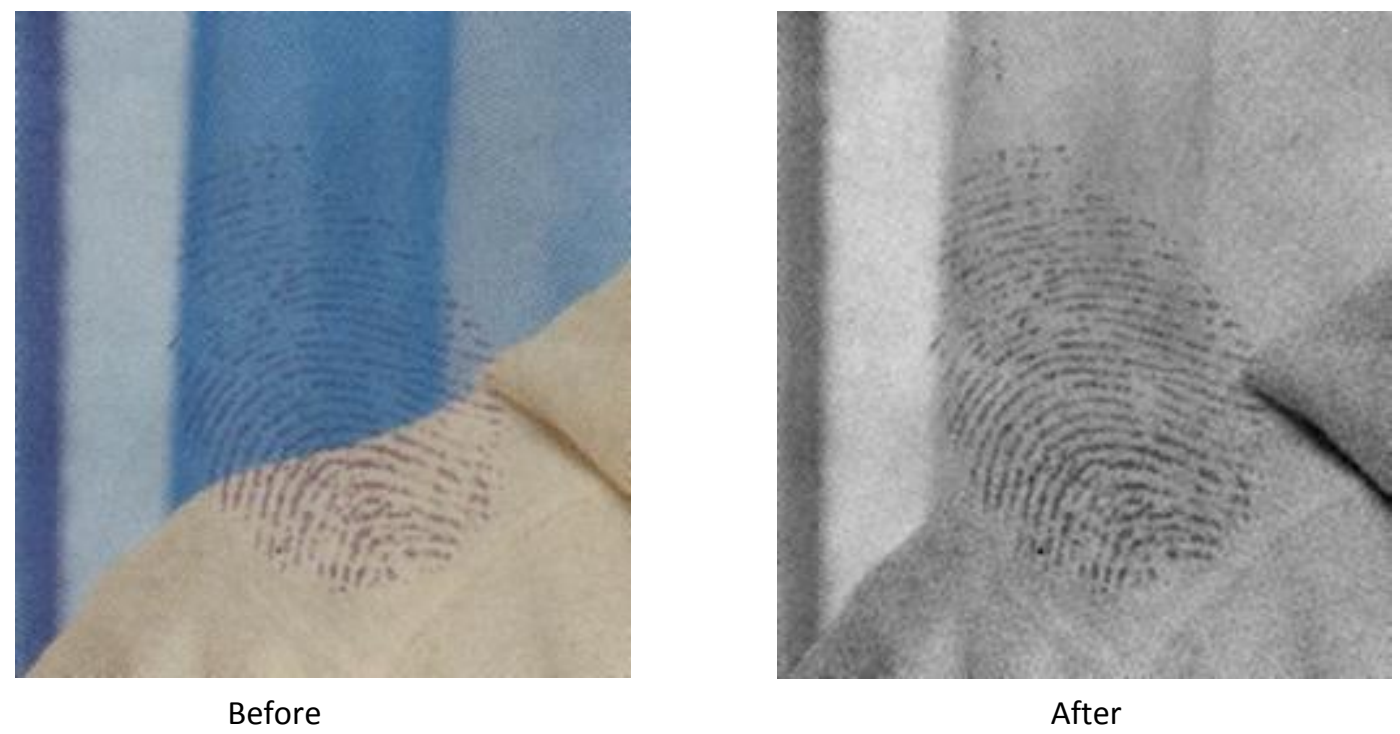

Figure 1: An example of forensic latent fingerprint preprocessing.

Clearly the fingerprint in the image after processing (on the right) visually exhibits more fingerprint pattern information. Figure 2 shows another example in which image preprocessing is essential. The image on the left shows a crime scene lift in which an impression is left on duct tape. The grid pattern of the duct tape is clearly visible in a green hue which, to the eye, completely masks fingerprint information. The image on the right shows the same latent print image after preprocessing. In this example, preprocessing included: developing using Basic Yellow (dye stain), color filtering using Channel ' $a$ ' in the Photoshop's Lab Color mode, application of a Fourier-based pattern removal filter (which is a product of Foray Technologies), and greyscale management with the Photoshop Levels command. The end result is a preprocessed image that clearly reveals ridge flow. 


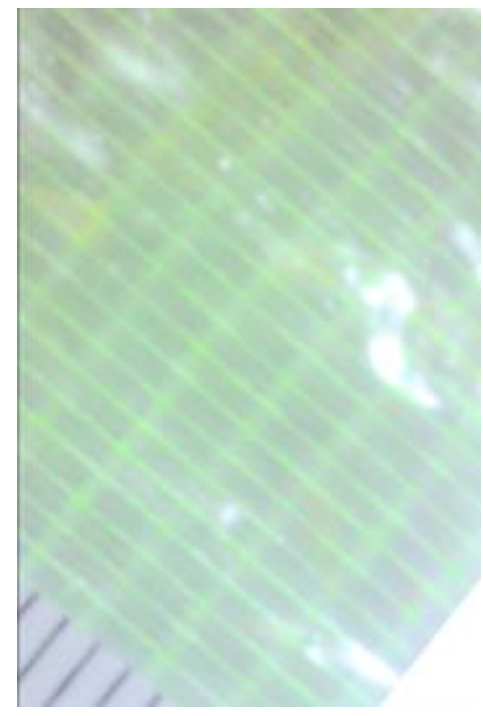

Before

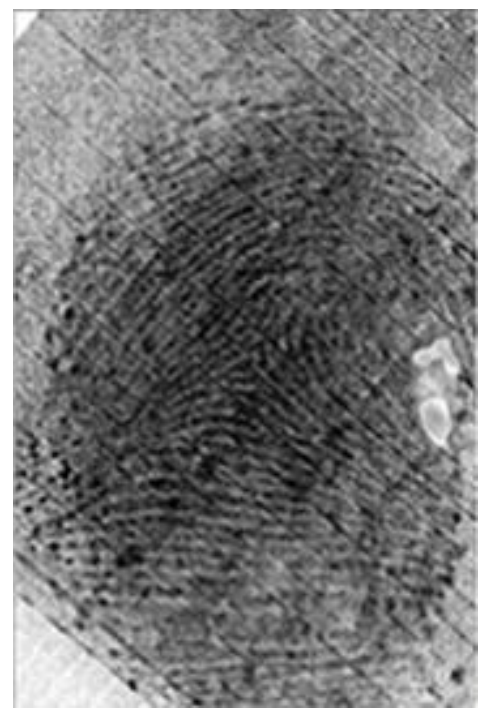

After

Figure 2: The necessity of latent fingerprint preprocessing.

The prevalence of latent fingerprint image preprocessing belies the fact that it is not a single activity but rather a complex process containing several sub-varieties. The preprocessing workflow is diverse in many aspects including: 1) the aggressiveness of the image preprocessing used to suppress various types of noise and, conversely, enhance fingerprint information; 2) the software tools that are used; 3) specific algorithms employed; and 4) operator-specific preferences. For one, different image software systems use different implementations of even the most basic image functions. See, for example, the multiple implementations of RGB-to-grey-scale conversation [3] [4]. Even more, the same fingerprint may be enhanced in different ways as fingerprint examiners may have their own analysis style. Finally, the desired endpoints of the preprocessing may be different, and matching systems can have distinct criteria for their input. Figure 3 shows an example of different endpoints for the same input image.

The overarching principle for image preprocessing is that the image transformations should neither add to, nor subtract from, fingerprint information contained within the image [24]. This guiding principle presently lacks analytical underpinnings. In [22], a crucial question was asked by the Scientific Working Group on Friction Ridge Analysis, Study and Technology (SWGFAST): "What methods can enhance images without risking bias in results (e.g., due to altering image)? (Question 1.3.1 in page 30)" Although there is some research on this topic, see for example, the "image processing" paragraph on page 34, and "Effects of development techniques" on page 35, universal standards of best practice are needed. 
The goal of this study is to characterize the effects of image preprocessing that transforms the latent fingerprint image obtained from the crime scene ('before image') to the image used for identity analysis ('after image').

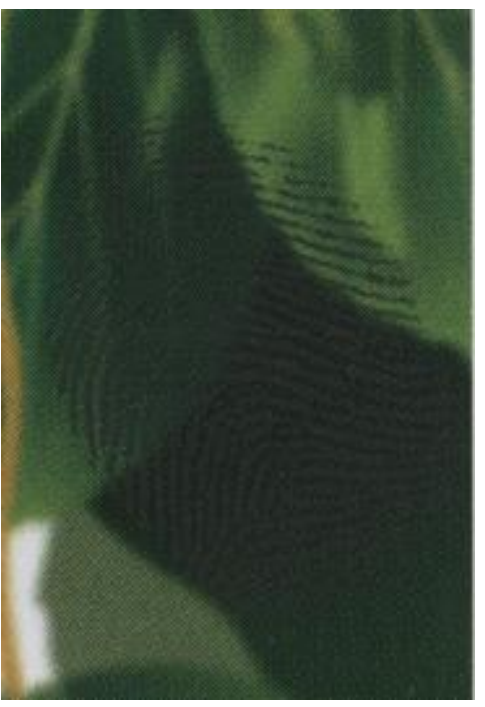

(a) Original latent fingerprint

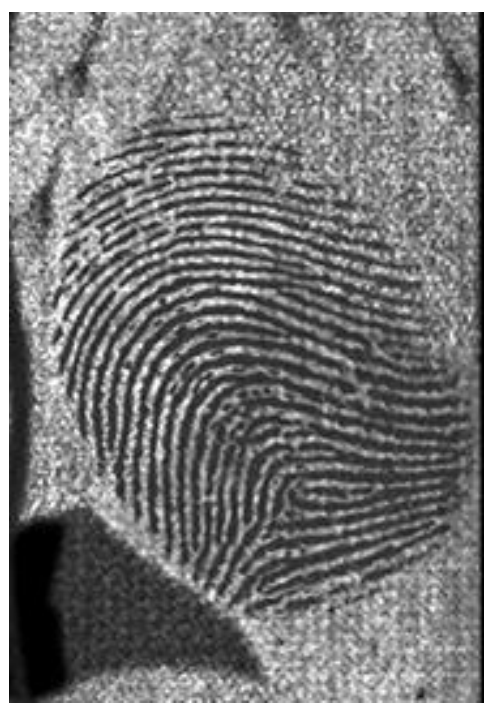

(c) After first preprocessing



(b) After second preprocessing

Figure 3: Distinct endpoints of forensic latent fingerprint preprocessing.

\subsection{THE SOFTWARE AND TOOLS FOR LATENT FINGERPRINT PREPROCESSING}

A number of image analysis tools are capable of revealing latent fingerprint information comingled with noisy background features. The range of applicable software includes: commercial photo editing tools designed for general purpose image editing such as Adobe Photoshop and Pixelmator; open source image editing tools such as the GNU Image Manipulation Program and Inkscape; scientific toolboxes such as the Matlab image processing toolbox and Open Source Computer Vision; and specialty software particularly designed for latent fingerprint analysis such as the FBI's Latent Fingerprint Services, Universal Latent Workstation, Automated Fingerprint Identification System (AFIS)'s interactive graphical user interface for latent prints, etc. Although there are general common functions in those software toolboxes, different software tools are designed for different purposes and used for different applications. Specifically for forensic fingerprint recognition, we need to define analysis and procedures to verify that the software follows the basic principle and requirements of latent fingerprint preprocessing, thereby ensuring the repeatability of the process and guaranteeing image integrity. 


\section{METHOD}

\subsection{LITERATURE REVIEW}

Although latent fingerprints are well and widely studied by forensic scientists [3], there has been little systematic analysis of forensic latent fingerprint image preprocessing. Digital image preprocessing is a relatively new area requiring research in order to establish its scientific foundations. It is especially critical to investigate the image transformations used in the course of this analysis, and to propose effective approaches or give general suggestions to guide the preprocessing workflow to improve the latent fingerprint quality and preserve the fingerprint integrity at the same time. The principle objective of this work is to initiate such investigations.

Latent fingerprint images are obtained under non-ideal acquisition conditions; the finger impression may be incomplete, distorted, or corrupted by background noise. In most cases, the latent fingerprint quality is crucial for latent identification. The research community has developed several approaches and algorithms on fingerprint image quality [11], [12], [14], [15] and latent fingerprint preprocessing [13]. In [14], a latent fingerprint image quality (LFIQ) measurement was proposed. In [13], Yoon, et al. proposed a latent fingerprint enhancement algorithm requiring a manually marked region of interest (ROI) and singular points. The paper proposed a novel orientation field estimation algorithm, which fits the coarse orientation map to an orientation field model. Experimental results on the NIST SD27 Latent Fingerprint Database [19] indicate that, with the use of the proposed enhancement algorithm, the matching accuracy of the commercial matcher, Rank-m identification Rate, was improved by $5 \%$ to $20 \%$.

\subsection{METHOD OVERVIEW}

We seek to compare the image qualities of before images - original RGB images, directly obtained from forensic crime scene photography - and after images - the grey scale image after preprocessing - to evaluate the performance of the preprocessing effect.

In the context of latent fingerprint analysis, a primary objective is to improve contrast between ridges and furrows, thereby enabling clearer identification of minutia points. The ridges and furrows appear as periodic structures in the fingerprint image. This periodicity manifests as narrow regions with relatively high-energy content in the frequency spectrum of the image. It is natural to construct a latent fingerprint quality metric built on this feature.

Our latent fingerprint quality metric is based on an extension of the Spectral Image Verification and Validation analysis (SIVV) [1]. The original SIVV algorithm was designed 
for image validation and verification of ten-print fingerprint images from live-scan devices, and for maintaining the fidelity of fingerprint image databases. SIVV can effectively differentiate the non-fingerprint input from the flat or rolled fingerprint input. As the periodic structure of the fingerprint ridges and furrows is a level one feature, SIVV is potentially applicable to the latent fingerprint preprocessing domain. However, latent fingerprint images are often corrupted by complex background noise, and the ridge structures may not be clearly visible. Furthermore, latent images are generally of poor quality and the fingerprints can be incomplete. These characteristics will confound the original SIVV feature analysis; refinement is needed for SIVV to be applicable to latent fingerprint images.

We implement several modifications to SIVV in light of the above-mentioned difficulties. In order to suppress confounding background noise, in the spatial domain the algorithm focuses analysis on a region of interest within the fingerprint image. Furthermore in the frequency domain, the algorithm constrains the SIVV peak to be within a limited range, which can be inferred by the fingerprint ridges' pixel distances on the latent fingerprint images. The resulting metric is still based on the intrinsic Fourier spectral properties of latent fingerprint images. The new latent fingerprint quality metric provides the quantitative measurement to characterize the quality of the latent fingerprint images and measures the effectiveness of the latent fingerprint preprocessing process.

\subsection{SIVV ON FLAT/TEN-PRINT OR ROLLED FINGERPRINT}

SIVV analysis derives from the periodicity of ridges and furrows [1]. For completeness, first we summarize the original SIVV algorithm (for the detailed presentation, please refer to the original report [1]).

\section{Step 1. Image Windowing}

The standard one-dimensional Blackman window is given in the following equation:

$$
\begin{gathered}
w(n)=0.42-0.5 \cos \left(\frac{2 \pi n}{N-1}\right)+0.08 \cos \left(\frac{4 \pi n}{N-1}\right) \\
\text { where } 0 \leq n \leq N-1
\end{gathered}
$$

The length of the one-dimensional window is $\mathrm{N}$. The constant numbers in the equation are the same as the standard Blackman filter. Given the image with $\mathrm{N}$ rows and $\mathrm{M}$ columns, the two-dimensional Blackman Window is the tensor product of windows of length $\mathrm{N}$ and $\mathrm{M}$. When the 2D Blackman Window is applied to the fingerprint image, the window is applied on the center of the fingerprint texture, and the size is adapted to the size of the fingerprint image. 
Step 2. Discrete Fourier Transform (DFT)

$$
H(u, v)=\sum_{x=0}^{M-1} \sum_{y=0}^{N-1} \exp \left[2 \pi i y \frac{v}{N}\right] \exp \left[2 \pi i x \frac{u}{M}\right] h(x, y)
$$

Here $u$ and $v$ denote frequency components in the $x$ and $y$ directions ranging from $\frac{-M}{2}$ to $\frac{M}{2}$ and $\frac{-N}{2}$ to $\frac{N}{2}$ respectively.

\section{Step 3. 2D (normalized) Log Power Spectrum}

The $2 \mathrm{D}$ power spectrum is computed as:

$$
P(u, v)=|H(u, v)|^{2}
$$

Depending on the implementation, the output of this step can be normalized or notnormalized; that is

$$
10 * \log P(u, v)
$$

Or

$$
10 * \log \frac{P(u, v)}{P(0,0)}
$$

$\underline{\text { Step 4. 2D Polar Transform of Power Spectrum }}$

The $2 \mathrm{D}$ power spectrum is represented in polar coordinates using the transformation:

$$
\begin{aligned}
& \rho=\frac{\sqrt{u^{2}+v^{2}}}{\sqrt{M^{2}+N^{2}}} \\
& \theta=\tan ^{-1}\left(\frac{v}{u}\right)
\end{aligned}
$$

where the $[0,0]$ point is in the image center. We use $P(\rho, \theta)$ to represent the $2 \mathrm{D}$ results of the polar transformation, where $\rho$ is divided by the maximum dimension of the input image $\mathrm{N}$, normalized to 0 and 0.5 cycles/pixel.

\section{$\underline{\text { Step 5. 1D Normalized Polar Transform }}$}

Finally, the 1D polar transform is computed as the sum over angles of: 


$$
\begin{gathered}
P(\rho)=\sum_{\theta=0}^{180} P(\rho, \theta) \\
\rho=0, \ldots, 0.5 \quad \text { cycles } / \text { pixel }
\end{gathered}
$$

The normalized 1D polar curve is:

$$
P_{N}(\rho)=\frac{P(\rho)}{P(0)}
$$

$$
\rho=0, \ldots, 0.5 \quad \text { cycles } / \text { pixel }
$$

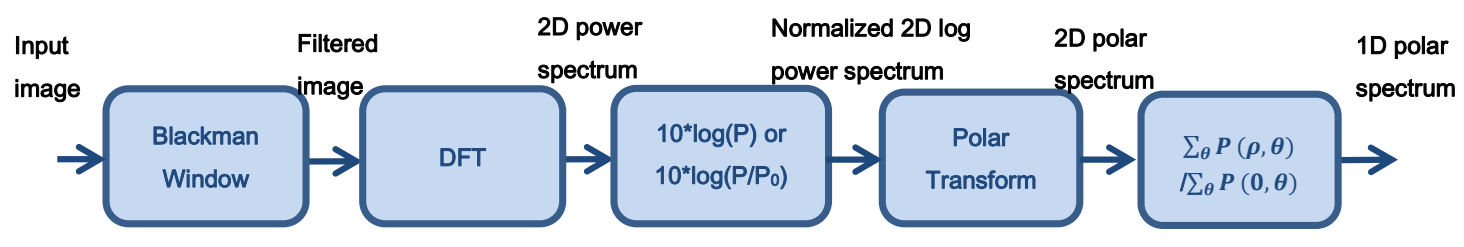

Figure 4: SIVV algorithm. 




(a) Original image



(e) $2 \mathrm{D}$ power spectrum

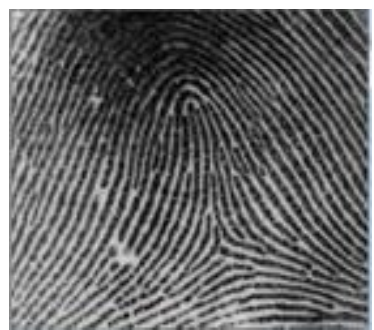

(b) Cropped image

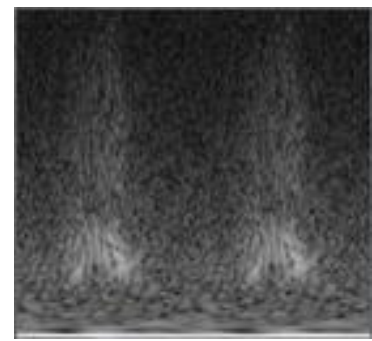

(f) 2D polar spectrum

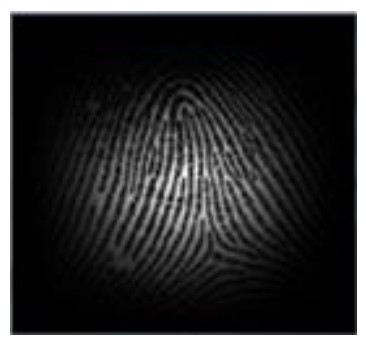

(c) After Blackman window

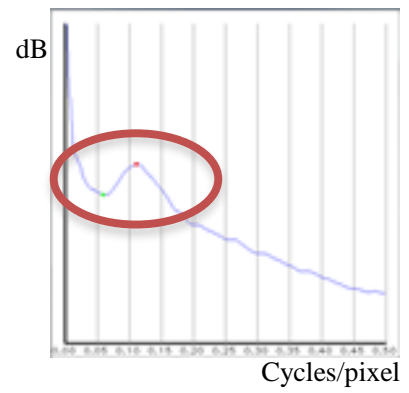

(g) 1D polar spectrum

Figure 5: An example of SIVV feature on a rolled fingerprint image.

Figure 4 shows the algorithm schematic. Figure 5 shows the analysis step-by-step. ${ }^{1}$ The clear peak and valley in the polar power spectrum (sub-figure $(\mathrm{g})$ ) is indicative of the ridgeflow periodicity and is referred to in the following as the SIVV feature.

The original objective of SIVV analysis is to screen fingerprint image databases for specimens improperly scanned from fingerprint cards [1]. In the auto-capture process, SIVV analysis can help to identify the auto-capture failures, identify non-fingerprint images that may have been incorrectly included in a fingerprint database, etc. The original SIVV algorithm focuses on the fingerprint datasets that were captured under controlled environments, such as flat/ten-print or rolled fingerprint database, or mixed database which contains face, iris and fingerprint, etc. Generally, the fingerprints in such datasets have clean background and significant less noise compared with the latent fingerprint image dataset.

${ }^{1}$ Courtesy of SIVV software package in NBIS [1] [20], the original image is G001T2U.tif in [19]. 


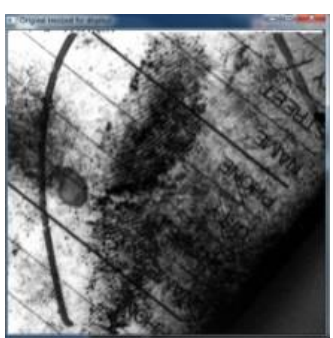

(a) Original image

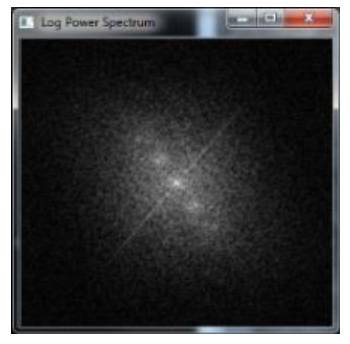

(d) 2D power spectrum

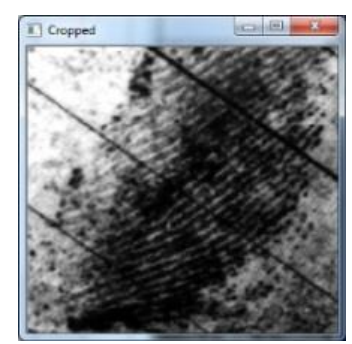

(b) Cropped image

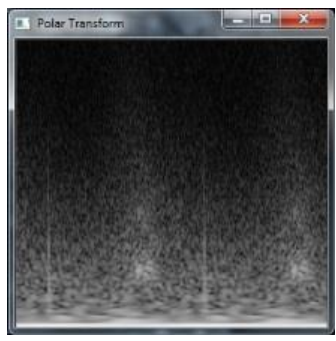

(e) $2 \mathrm{D} \log$ polar spectrum

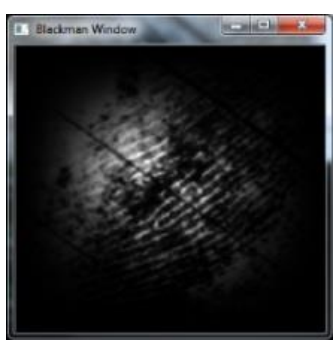

(c) After Blackman window

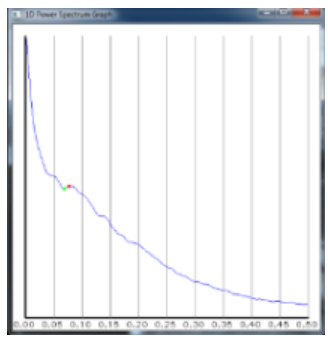

(f) $1 \mathrm{D} \log$ polar spectrum

Figure 6: An example of the SIVV feature on a latent fingerprint image.

\subsection{MOTIVATION OF OUR APPROACH}

The original SIVV feature performs well on the flat/ten-print or rolled fingerprint database, which are captured by inking methods or live-scan devices in an attended mode. In such contexts, background noise is minimized during the capture, and the contrast between the ridges and furrows is relatively high. As image quality is generally controlled very well, the fingerprint image ridges and valleys are clear and computer-readable. In such cases, the periodic structure of the ridges and valleys can be captured by Fourier spectrum analysis in the frequency domain. It follows that SIVV performs well when the original fingerprint image is of good quality.

In comparison with rolled fingerprints, original latent fingerprint images are of much poorer quality in several respects. Latent fingerprints are generally smudgy and blurred. They often capture only a small finger area, and may have large nonlinear distortion due to pressure variations. Finally, and perhaps the most damaging source of noise from the perspective of SIVV analysis, in latent fingerprint images, it is not uncommon for the fingerprint image to be superimposed on a structured background. This background "noise" is unavoidable and is extremely hard to model due to the large variety of background colors, textures, etc. When background noise is strong, the spectral spike from the fingerprint periodicity can be conflated with the signals of other periodic structures of the images. Sources of such patterned noises are diverse and include textile fabric, written text, and residue from the 
physical processing. Figure 6 shows an example ${ }^{2}$ of original SIVV on a latent fingerprint image in the NIST Database 27 [19]. It shows that the SIVV spike is largely weakened due to the fingerprint incompleteness and from being submerged in the background noise.

Figure 7 (implementation courtesy of [20]) shows the results of applying the original SIVV analysis [20] to six unprocessed, latent fingerprint images taken from a database of training images provided by Schwarz Forensics and Foray Technologies (the image database is described in more detail in 3.1). These images demonstrate that the original SIVV analysis may not be directly applicable to latent fingerprint images. In the cases $1,3,5$, and 6 , there is no obvious spike (fingerprint level-one information) at all. In cases 2 and 4, the detected peak (red arrow) does not represent the fingerprint ridges and furrows, but rather arises due to texture of the background. In addition, 1-c and 2-c show the detailed texture noise (tiny grids) on the images. The actual fingerprint ridge peak is shown by the green arrows in 2-b and 4-b.

In this report, we introduce two additional components to the SIVV analysis to regain the valuable metrical functionality that is otherwise lost in latent fingerprint contexts. First, we introduce a Region of Interest (ROI) selection to enable the analysis to focus only on a local subregion which contains fingerprint signal in spatial domain. Second, we introduce a constraint in the spectral analysis restricting attention to a small window which may contain the ridge and furrow spike in the frequency domain.

2 Courtesy of SIVV software package in NBIS [1] [20], the original image is G001L2U.tif in [19]. 


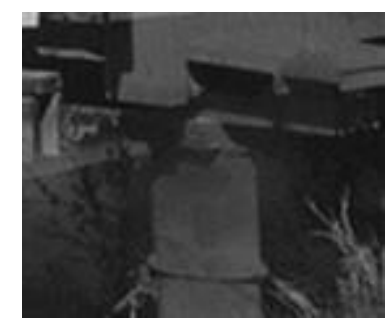

1-a. Original image (158)



2-a. Original image (165)

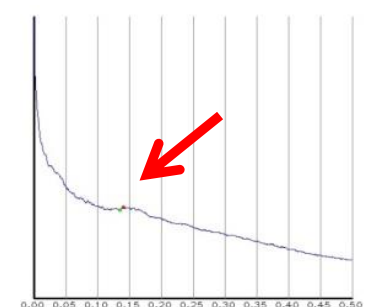

1-b. 1D log polar spectrum

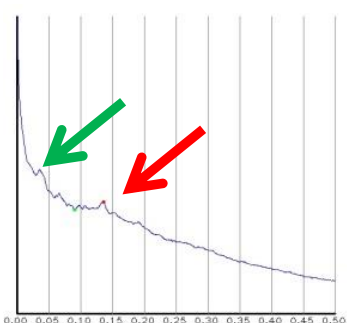

2-b. 1D log polar spectrum

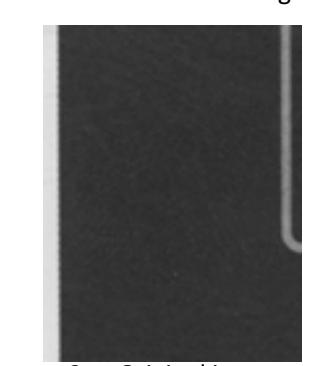

3-a. Original image

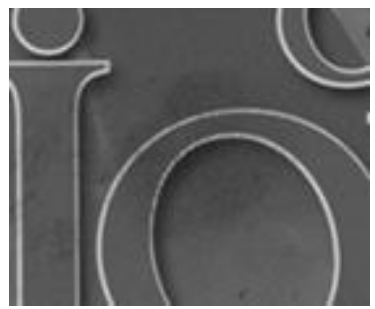

5-a. Original image (160)

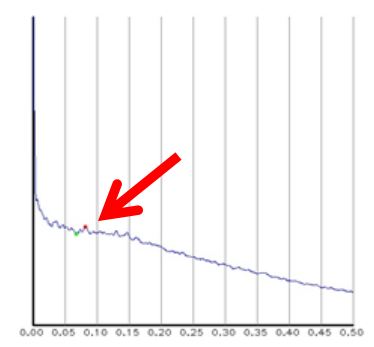

3-b. $1 \mathrm{D}$ log polar spectrum

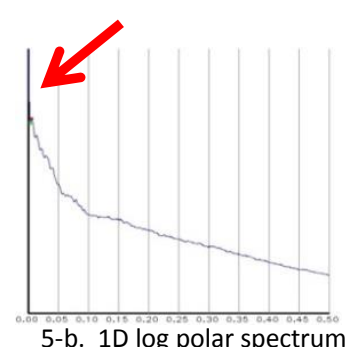

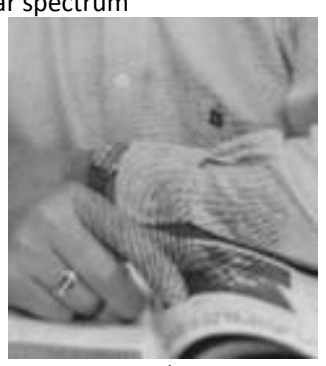

4-a. Original image

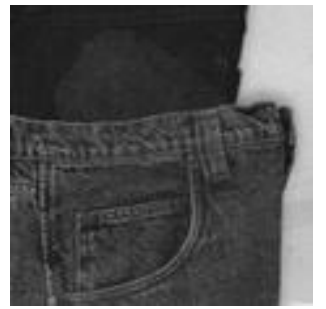

6-a. Original image

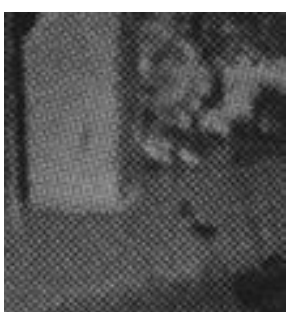

1-c. Zoom in the background noise of (2-a)

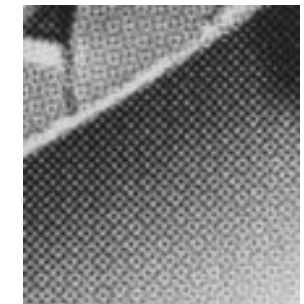

2-c. Zoom in the background noise of (3-a)

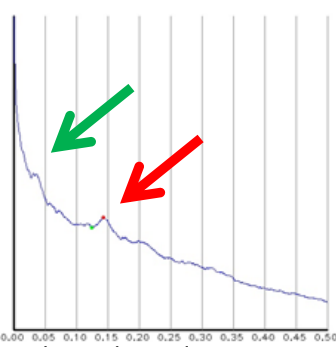

4-b. 1D log polar spectrum



Figure 7: Examples of the SIVV feature on latent fingerprint images.

\subsection{BLACKMAN WINDOW}

In fingerprint analysis, the Blackman window is used to suppress signal outside of the fingerprint region in addition to eliminating non-periodic boundary effects. The standard 1D Blackman Window is defined by:

$$
w(n)=\frac{1-\alpha}{2}-\frac{1}{2} \cos \left(\frac{2 \pi n}{N-1}\right)+\frac{\alpha}{2} \cos \left(\frac{4 \pi n}{N-1}\right)
$$


where $0 \leq n \leq N-1$ and $\alpha=0.16$. The resulting curve is shown in (Figure 8). The 2D Blackman window filter is the cross product of two 1D Blackman window vectors ( $\mathrm{N}$ maybe different in two 1D vectors).

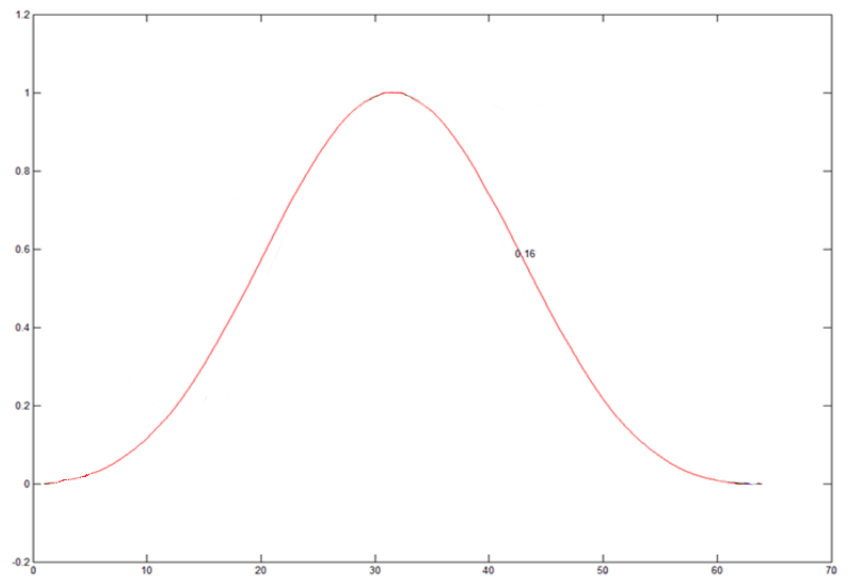

Figure 8: Blackman Window $(\alpha=0.16)$.

In latent images, variability in fingerprint location, orientation, and size requires that we add more flexibility in application of the Blackman window filter to the image. We include additional parameters to control the location (center point of the 2D filter), size (if the fingerprint region is modeled by an ellipse with the ellipse's major axis of $N_{\max }$ pixels and minor axis of $N_{\min }$ pixels respectively, the size can be controlled by using $N_{\max }$ and $N_{\min }$ in the Blackman window equation), and orientation (the filter can be rotated in 2D plane) of the Blackman window. The constant numbers in the Blackman equation control the shape of the filter. To be faithful to the original Blackman filter, we keep the constant number unchanged and the curve shape unchanged. In practice, in order to increase the SIVV signal strength, we like to focus only on the ridge furrow patterns by customizing the size of the filter window to the size of the fingerprint area, and aligning the Blackman filter center to the fingerprint center. Figure 9 contrasts the following SIVV results: no Blackman window (a); original Blackman window (b); and customized Blackman window (by customizing the size, aligning the location and orientation) (c). We see that the regular Blackman window does help to strengthen the fingerprint signal in some cases. The customized Blackman Window has more flexibility to select the best region, which is very useful to latent fingerprint image analysis as location, orientation, and extent of the print region vary greatly in latent fingerprint images. 

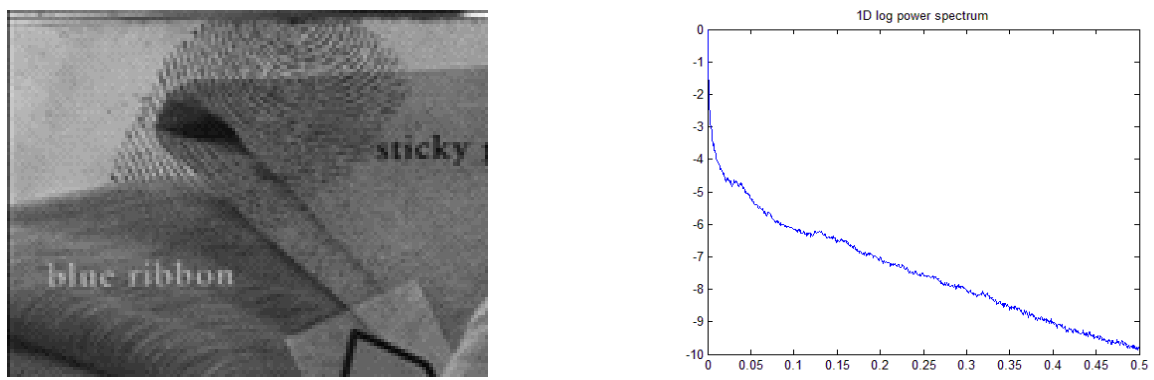

(a) a-1. Original image without Blackman Window (051e)

a-2.SIVV on whole image

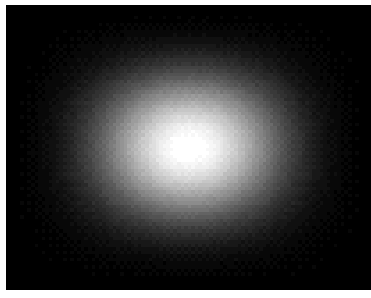

(b) b-1.Blackman Window

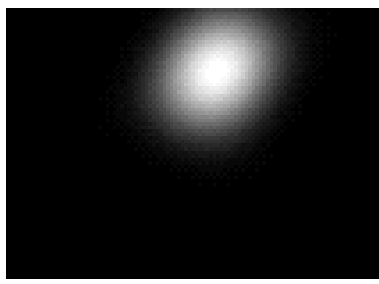

(c) c-1. Customized Blackman Window

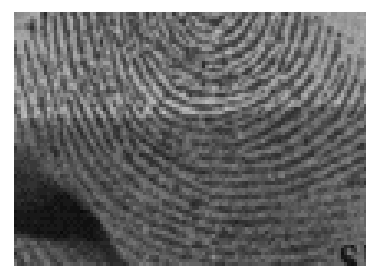

(d) d-1. ROI image [698 621519 589]

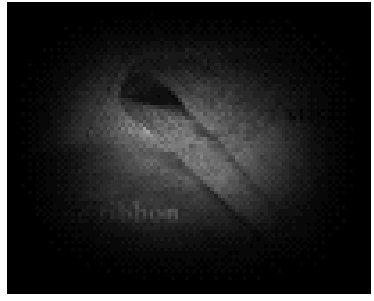

b-2. Blackman window on whole image

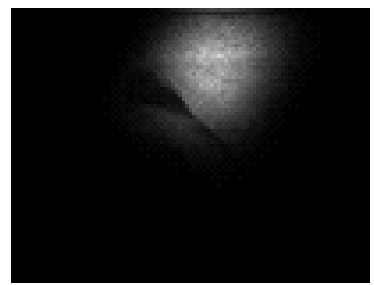

c-2. Image after customized Blackman Window

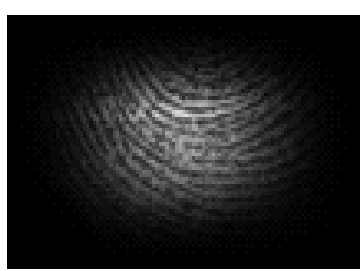

d-2. Blackman window on ROI image
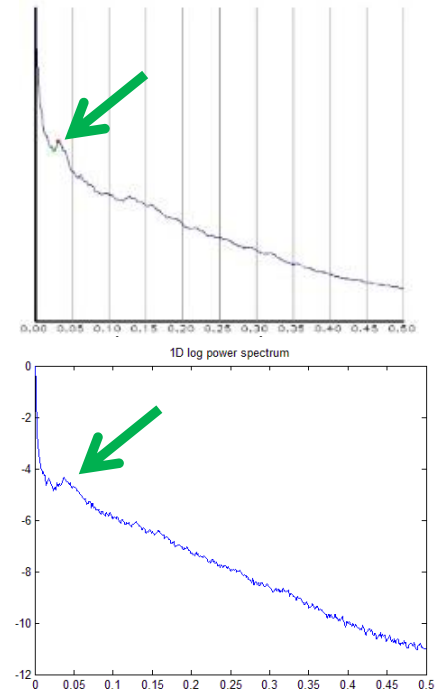

c-3. SIVV on c-2

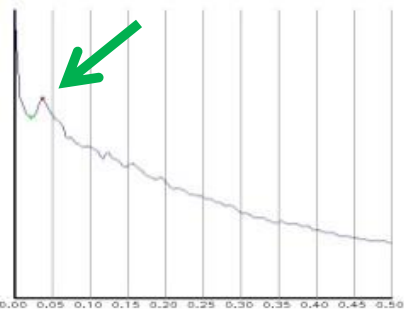

d-3. SIVV on d-2

Figure 9: The comparison of SIVV curves with different input options. 


\subsection{REGION OF INTEREST (ROI)}

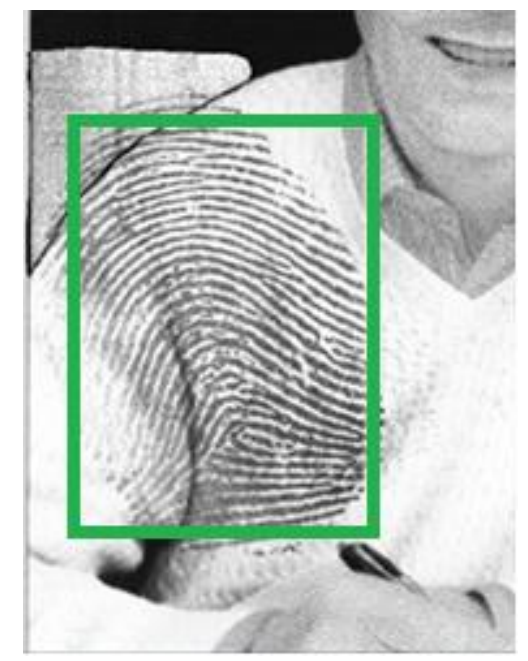

Figure 10: The selection of Region of Interest.

Latent fingerprint images often include significant noise (where noise is defined as any image feature that is not clearly identifiable as fingerprint information). Noise that overlaps with fingerprint region is intrinsically harder to diminish while simultaneously maintaining fingerprint integrity. One exception to this is when the noise is well separated from fingerprint information in a color space, in which case color filtration can be a powerful and effective tool. In general, such separation is not the case. For this reason, it is important that our analysis be designed with a high degree of specificity to fingerprint information. By contrast, it is relatively easy to remove the background noise in the area outside of the fingerprint region. Furthermore, it is important to do so as these regions contain no fingerprint information yet, due to the nonlocal nature of Fourier-based spectral analysis, they may contribute a large amount of non-signal energy, thereby masking the fingerprint SIVV feature in the frequency domain. Thus we specify region of interest (ROI) by selecting a rectangular region containing the entire fingerprint. An example is shown in Figure 10. Image features outside the ROI are masked by setting the intensity value to a constant black. This ROI selection is done prior to the FFT step of the SIVV and performed "in place," i.e., the image remains the size of the original, so as to maintain pixel density and not introduce rescaling artifacts. During the latent fingerprint image quality measurement process, there is a trade-off between the fingerprint region size and fingerprint region purity. The ROI should contain most of the $\operatorname{area}^{3}$ of the fingerprint image with good ridge information. Currently the

\footnotetext{
${ }^{3}$ Notice that the ROI proposed here is only for latent fingerprint detection and quality measurement, not for latent fingerprint identification. The whole fingerprint region should be considered in the identification process.
} 
rectangle shape ROI selection must be done manually. In the future, we may consider a semiautomatic ROI extraction method perhaps based on an elliptical or polygon region.

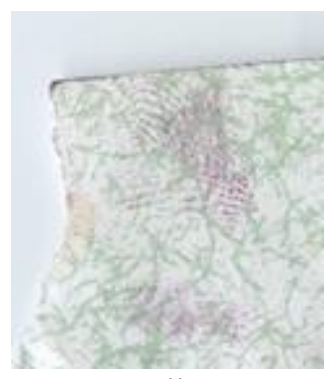

Full Image

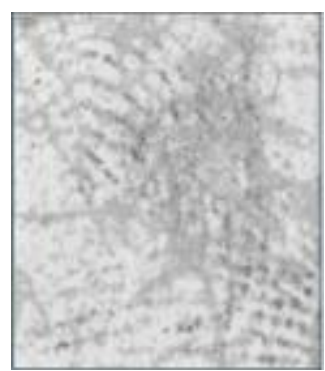

Region of Interest

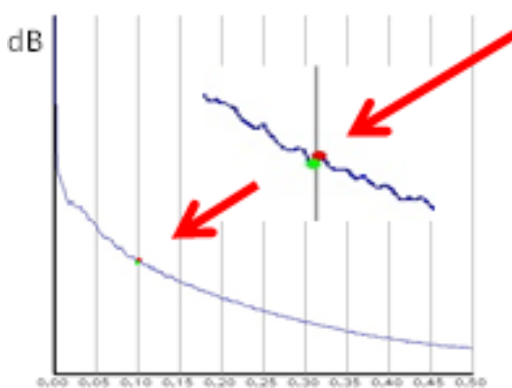

Cycles/pixel

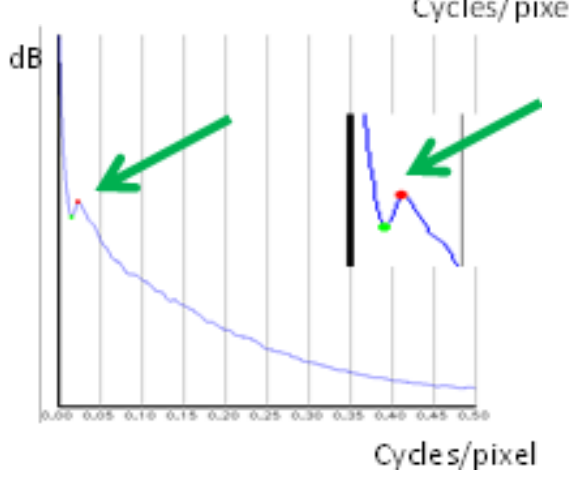

Figure 11: An example of Region of Interest.

Figure 11 shows the comparison of SIVV curves using the whole image and the ROI. The SIVV spike (indicated with arrow) of the ROI image is much stronger than the SIVV spike of the whole image. The example shows that focusing on the ROI helps to recover the SIVV feature from the background noise.

The signal strength of the SIVV peak is predominantly determined by two factors. The first is the frequency power of the finger print ridge and furrow structure. This power is related to the area size of the fingerprint region; that is, the larger area includes more ridges and furrows and thus the stronger frequency power. The second factor is the signal-tobackground noise ratio (SNR). Here also, the larger the SNR, the stronger the SIVV signal peak. In summary, when determining the actual fingerprint ROI image, one must implement a trade-off between the size of the fingerprint region and the signal/noise ratio inside this region. Choose too small a region and the SIVV signal will be weak; choose too large a region such that it includes background noise and the SIVV signal will be buried. Figure 11 demonstrates that if we use full image as input, the fingerprint signal is submerged in the background noise. However, with appropriate selection of ROI, the fingerprint signal is detected in the SIVV curve. 


\subsection{PEAK LOCATION CONSTRAINT}

Research has demonstrated that frequency-based filtering can be an effective way to suppress background interferences associated with fingerprint evidence [14], [17]. In the SIVV computation, a 2D-Fast Fourier transform (FFT) is computed to extract the frequency information associated with an image. The 2D Fourier spectrum represents both power spectral density and phase information. Under favorable circumstances, the frequencies associated with the friction ridge detail in the print will be separable from those frequencies associated with the interfering background features. Selective filtering of frequencies associated with fingerprint information may filter out the background interference and correctly locate the SIVV peak.

The distance between fingerprint ridges is generally distributed over a narrow range. The study of [18] reports that the distance between ridges "ranged from $0.2 \mathrm{~mm}$ to $0.85 \mathrm{~mm}$ on fingerprints of male subjects and from $0.2 \mathrm{~mm}$ to $0.75 \mathrm{~mm}$ on fingerprints from female subjects. The mean ridge-to-ridge distance for 731 measurements on the male subjects was $0.46 \mathrm{~mm}$. In 1046 measurements on the female subjects, the mean value was $0.41 \mathrm{~mm}$." If the image resolution of the fingerprint image is known, then the distances between ridges measured in pixels may be estimated. The location of the SIVV fingerprint peak directly related to the repetitive ridge pattern and the ridge distance in pixels, can furthermore be calculated from the physical ridge distance range and the image resolution. See Figure 14 in NIST report [1], which shows the distribution of frequency location of SIVV feature for nonfingerprint image and fingerprint image in a mixed image dataset. It demonstrates the concept: for fingerprint images with 500 ppi resolution, most of the SIVV peak locations are between 0.01 to 0.15 cycles per pixel. Given 0.01 cycles per pixel is equal to $0.01 \times 500 / 25.4$ cycles per millimeter, which is about 0.197 cycles per millimeter (where 1 inch $=25.4$ millimeter, the image resolution is 500 pixels per inch). 0.01 to 0.15 cycles per pixel is equivalent to 0.197 to 2.95 cycles per millimeter; the mean of the peak location is around 0.08 cycles per pixel (about 1.57 cycles per millimeter). The same results are also shown in Appendix C, Figure C-5 and C-6 in the NIST report [1]. The two figures show the distributions of frequency location of SIVV peak for SD27 and SD29 dataset sampled at 500 ppi respectively. The peak locations consistently fall in the similar range between 0.01 to 0.15 cycles per pixel ( 0.197 to 2.95 cycle per millimeter) and the mean of the peak location is also around 0.08 cycles per pixel (1.57 cycles per millimeter). More precisely, the peak range for the SD27 and SD29 datasets concentrates between 0.05 to 0.12 cycles per pixel (about 0.98 to 2.36 cycle per millimeter), and the mean of the peak location is around 0.08 to 0.09 cycles per pixel (about 1.57 to 1.77 cycles per millimeter). Figure C-4 in [1] also shows the similar concept for fingerprint images in SD27 with 1000 ppi resolution: the peak location range is between 0.025 to 0.06 cycles per pixel, which is also about 0.98 to 2.36 cycles per millimeter, and the mean is around 0.04 cycles per pixel (which is also about 1.57 cycles per millimeter). The range value in cycles/mm in Figure 14 of NIST report [1], Figure C-4, C-5, and $\mathrm{C}-6$ in [1] are consistent (0.197 2.95 cycles per millimeter) despite the image resolution. 


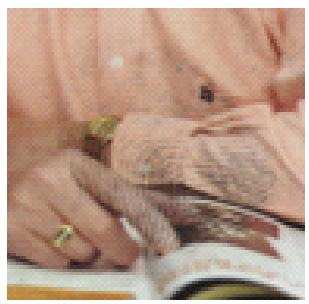

1. Original (153)



4. Enhanced (153)

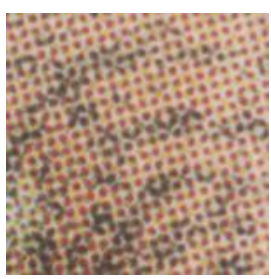

2. Zoom in

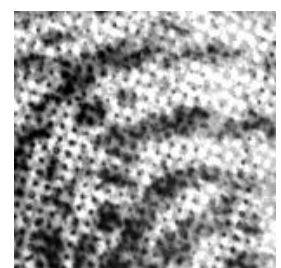

5. Zoom in on enhanced image



3. ROI

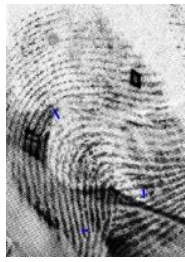

7. ROI

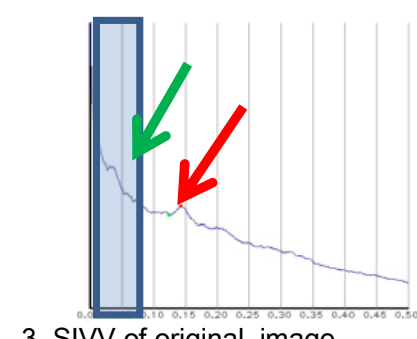

3. SIVV of original image

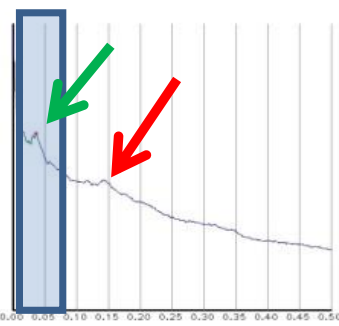

6. SIVV of the enhanced image

Figure 12: An example of SIVV peak location constraint.

The peak location can be estimated given the image resolution and the range of ridge distance. In practice, if there is a peak that is well outside this range, one may assume that the feature is generated by the background texture instead of the fingerprint (as shown by example 2 and 4 in Figure 7). In this manner, one can selectively filter out the background interference to remove the fake peaks and correctly locate the SIVV peak.

Figure 12 shows an example where the original image SIVV spectrum is shown in the first row, and the enhanced image spectrum is shown in the second row. The strongest peak in the original image is around 0.15 cycles per pixel (about 7.09 cycles per millimeter) in 1200 ppi images (the red arrow in Figure 12-3). By zooming in and looking closely at the details on the image in Figure 12-2, we can clearly see the grid texture in the background. The strongest peak around 7.09 cycles per millimeter is not a fingerprint peak but rather is derived from the frequency of the background texture, whose peak location is much greater than the fingerprint peak's upper bound (2.95 cycles per millimeter). The actual SIVV peak is the weak peak in the blue bar (the green arrow in Figure 12-3), which is around 0.035 cycles per pixel ( 0.689 cycles per millimeter) and in the range of fingerprint peak location ( 0.197 to 2.95 cycles per millimeter). The second row in Figure 12 confirms the analysis. After preprocessing, the fingerprint signal is strengthened and the background noise is weakened. In this case, the SIVV peak located inside the predicted bar is the strongest peak while the background texture's peak is weakened and becomes smaller. In summary, we can define the peak location constraint; that is, the approximate range for SIVV peak of the fingerprint with 
1200 ppi resolution is 0.197 to 2.95 cycles per millimeter, which is 0.004 to 0.062 cycles per pixel $^{4}$ and indicated by the blue bar in Figure 12.

\subsection{SOME PROPERTIES OF THE SIVV}

We draw attention to the invariance of the SIVV feature with respect to image noise and a common preprocessing image transformation. More exhaustive analysis of the propagation of SIVV analysis through a wider variety of preprocessing transformations and image noise models is a topic for future research.

\subsubsection{Image inversion}

Latent fingerprint examiners generally prefer that fingerprint information appear as black ridges as our eyes are better at picking out slightly off-white colors on a white background than distinguishing less black pixels on a black background. Figure 13 shows the image developed by white powder techniques and its invert image. It shows that normally human eyes are more sensitive to the black ridges in white background. Thus, for the latent fingerprint images which are developed by white powder techniques, generally it is preferred to invert the image to change it to black ridges with a white background. The operation of invert is:

$$
I_{\text {invert }}=255-I_{\text {original }} \text { for all pixels in } I_{\text {original }}
$$

\footnotetext{
${ }^{4} 0.197$ cycles per millimeter $=0.197 \times 25.4 / 1200$ cycles per pixel $=0.004$ cycles per pixel .
} 




1. Original image

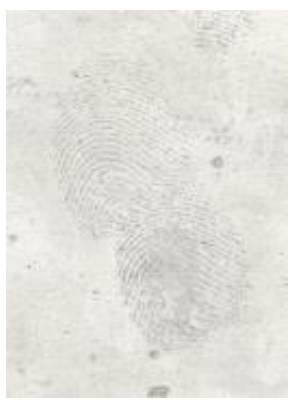

6. Invert image

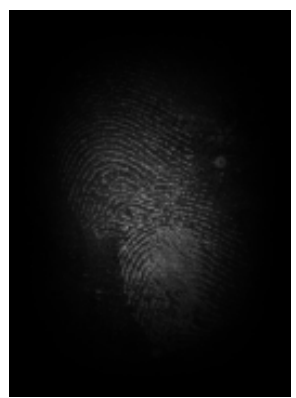

2. Blackman window on original image

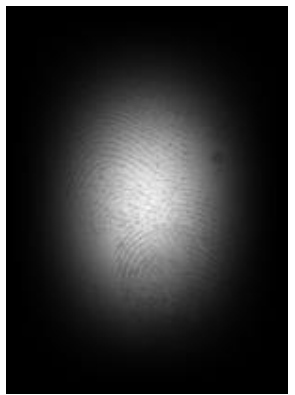

7. Blackman window on original image

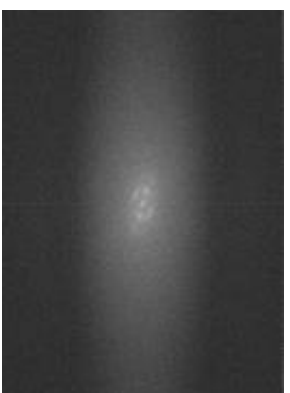

3. Power spectrum of original image



8. Power spectrum of original image

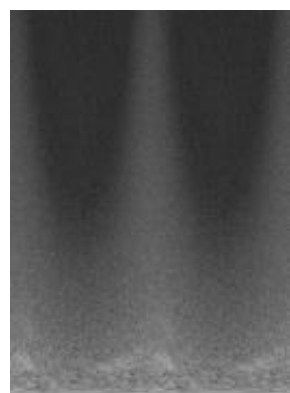

4. Polar spectrum of original image

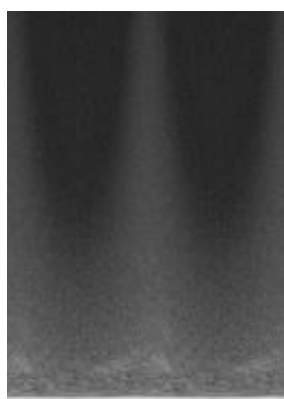

9. Polar spectrum of original image

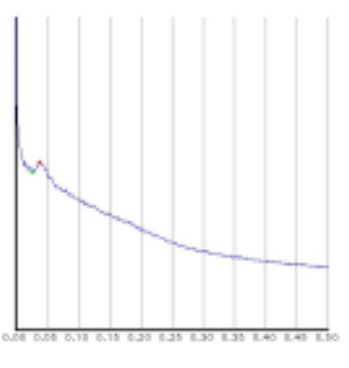

5. SIVV of original image

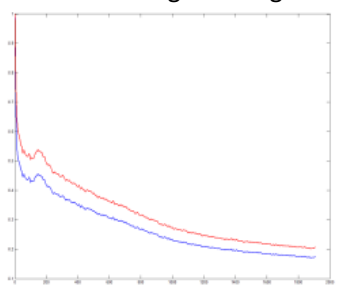

11. Red: original, Blue: Invert

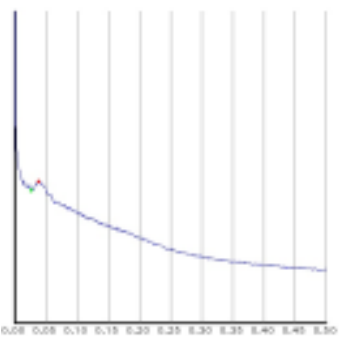

10. SIVV of invert image

Figure 13: The original image and its invert image of white power developed prints.

Although the image and its invert are quite different to human eyes, the invert operation does not add or remove any information. It is just a different way to represent the information. Thus, if we measure the fingerprint using the machine algorithms, both cases may provide the similar results except that the invert operator adds a power spectrum with a peak in the center $(0,0)$ and zero otherwise. In our experiments, we found that the metric curve of the invert image is very similar to the metric curve of its original image. Figure 14 shows an example. The SIVV curve nicely preserves the fingerprint ridges information in the invert image cases. 


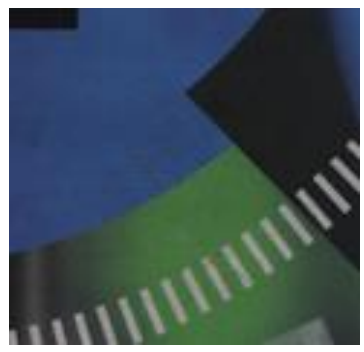

1. Original image

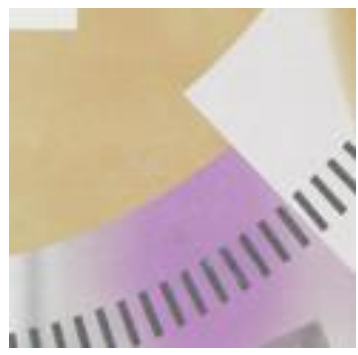

6. Invert image

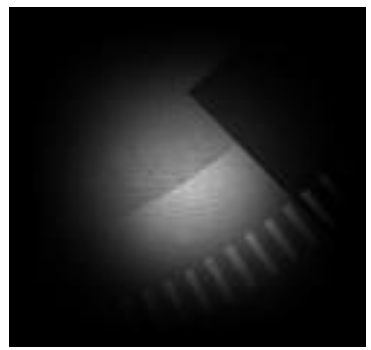

2. Blackman window on original image

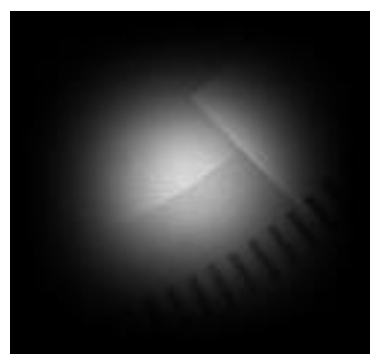

7. Blackman window on original image

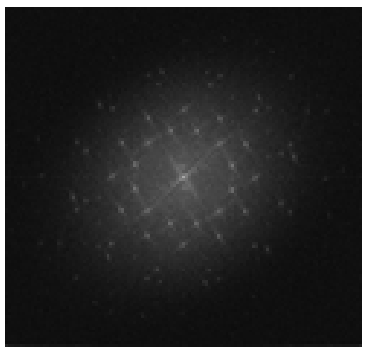

3. Power spectrum of original image

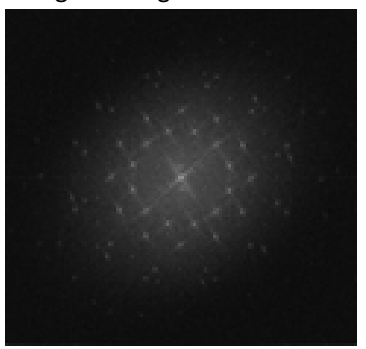

8. Power spectrum of original image

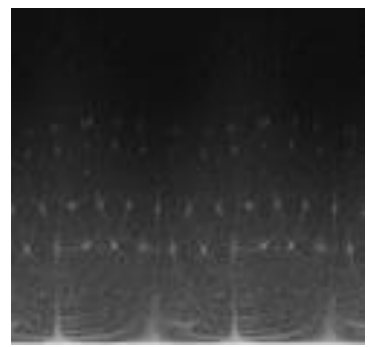

4. Polar spectrum of original image

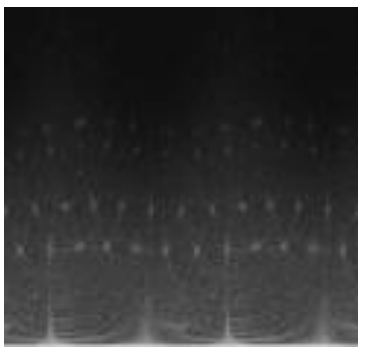

9. Polar spectrum of original image

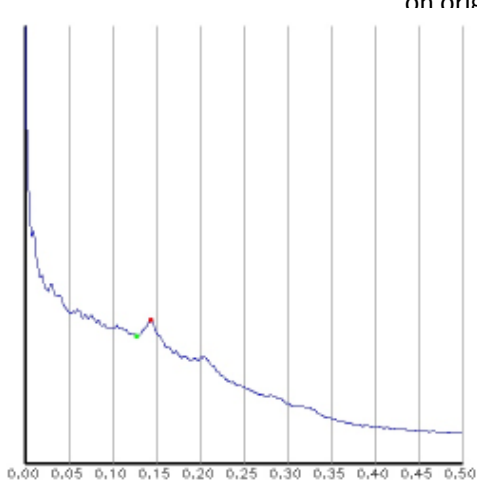

5. SIVV of original image



10. SIVV of invert image

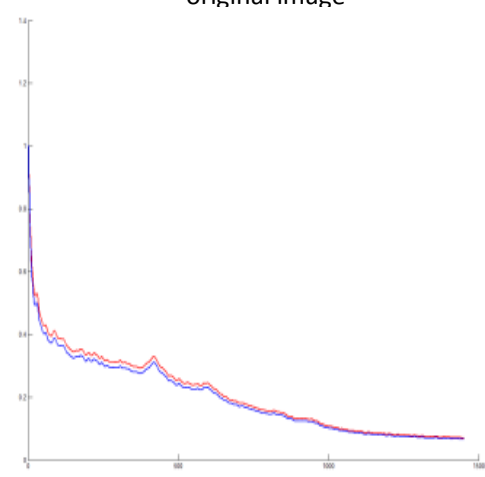

11. Red: original, Blue: Invert

Figure 14: The comparison of SIVV curves of an image and its invert image.

We can estimate the difference of the metric curves between the original image and its invert image in a mathematical way. Following the definition of SIVV given in Section 2.1 without considering the Blackman window filter (which is an optional step), according to the next four steps of SIVV computation, we have the relationship of the intermediate results of the original image and the invert image in each step:

(1) According to the linearity property of the DFT, we have:

$$
\begin{array}{r}
D\left[I_{\text {invert }}(x, y)\right]=D\left[255-I_{\text {original }}(x, y)\right]=D[255 * \text { ones }(M, N)]-D\left[I_{\text {original }}(x, y)\right] \\
=\text { A matrix with a value in }(0,0), \text { and } 0 \text { for others }-D\left[I_{\text {original }}(x, y)\right]
\end{array}
$$


(2) The 2D power spectrum is the magnitude of the DFT results. Without normalization on the signal, the original and invert image's 2D power spectrum is the same except in the origin point.

(3) The $2 \mathrm{D}$ polar spectrum is the coordinate transformation of the $2 \mathrm{D}$ power spectrum.

(4) The 1D polar is the sum in $\theta$ direction, and normalized by $P_{o}(\rho)$.

Thus, assuming that the ROI and Blackman Window are unchanged, we observe that the SIVV curves of the original image and its inversion are identical except at the origin point. After normalization and taking the logarithm, this difference manifests as an elementary shift of the SIVV curve up or down.

\subsubsection{SIVV on noise images}

We also test the SIVV analysis for robustness to independent identically distributed (IID) random noise. The Matlab function, $\mathrm{R}=\operatorname{randi}(\operatorname{IMAX}, \mathrm{N})$, is used to generate the pseudorandom integers from a uniformed discrete distribution, and it returns a $\mathrm{N}$ by $\mathrm{N}$ matrix containing pseudorandom integer values drawn from the discrete uniform distribution on [-IMAX: IMAX]. The Matlab codes for image generation are:

$\%$ I is the input image, magnitude is the noise level, and I_Noise is the output image.

$r=\operatorname{randi}([(-1) *$ IMAX, IMAX $]$, size $(I))$

I_Noise $=\operatorname{double}(I)+r$;

I_Noise $=$ uint8(I_Noise);

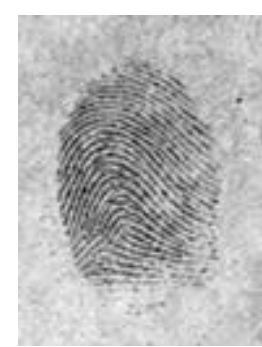

1. Original image

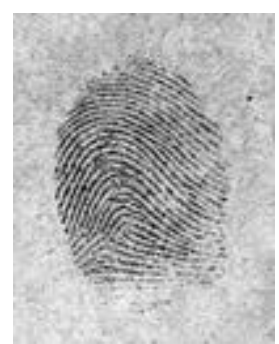

2. $\operatorname{IMAX}=3$

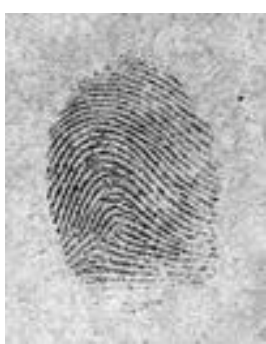

3. $I M A X=10$



4. IMAX $=50$

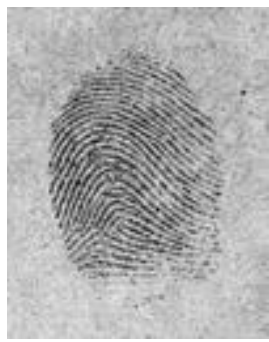

5. $I M A X=100$

Figure 15: The image with different levels of IID random noise.

Note, in the above Matlab code, uint8(X) converts the elements of the array $\mathrm{X}$ (here, $\mathrm{X}$ is I_Noise) into unsigned 8-bit integers. $X$ can be any numeric object, such as a DOUBLE. The values of a uint 8 range from 0 to 255 . Values outside this range saturate on overflow, namely they are mapped to 0 or 255 if they are outside the range.

We performed an experiment on the comparison of SIVV curves of an image with different levels of IID random noise without considering the Blackman window filter (which is an optional step). Figure 15 shows the initial image and the images by adding different random noise on it. The pictures shown are blurred due to Microsoft Word image compression. To 
the eye, at the resolution shown, the images in Figure 15 may appear identical. The highfrequency noise implied by our IID noise model can potentially affect the analysis. Figure 16 shows a portion of the IID random noise matrix in the experiment that added noise to the original image. Figure 17 shows the results of the SIVV curves by adding different levels of noise to the same image. It shows that the peak locations' $x$ coordinates are the same and the SIVV peaks' shapes are very similar. The SIVV curves are similar but the tails vertically move up along the $\mathrm{Y}$ axis as the noise magnitude increases. If we add more noise, the tail is even higher.

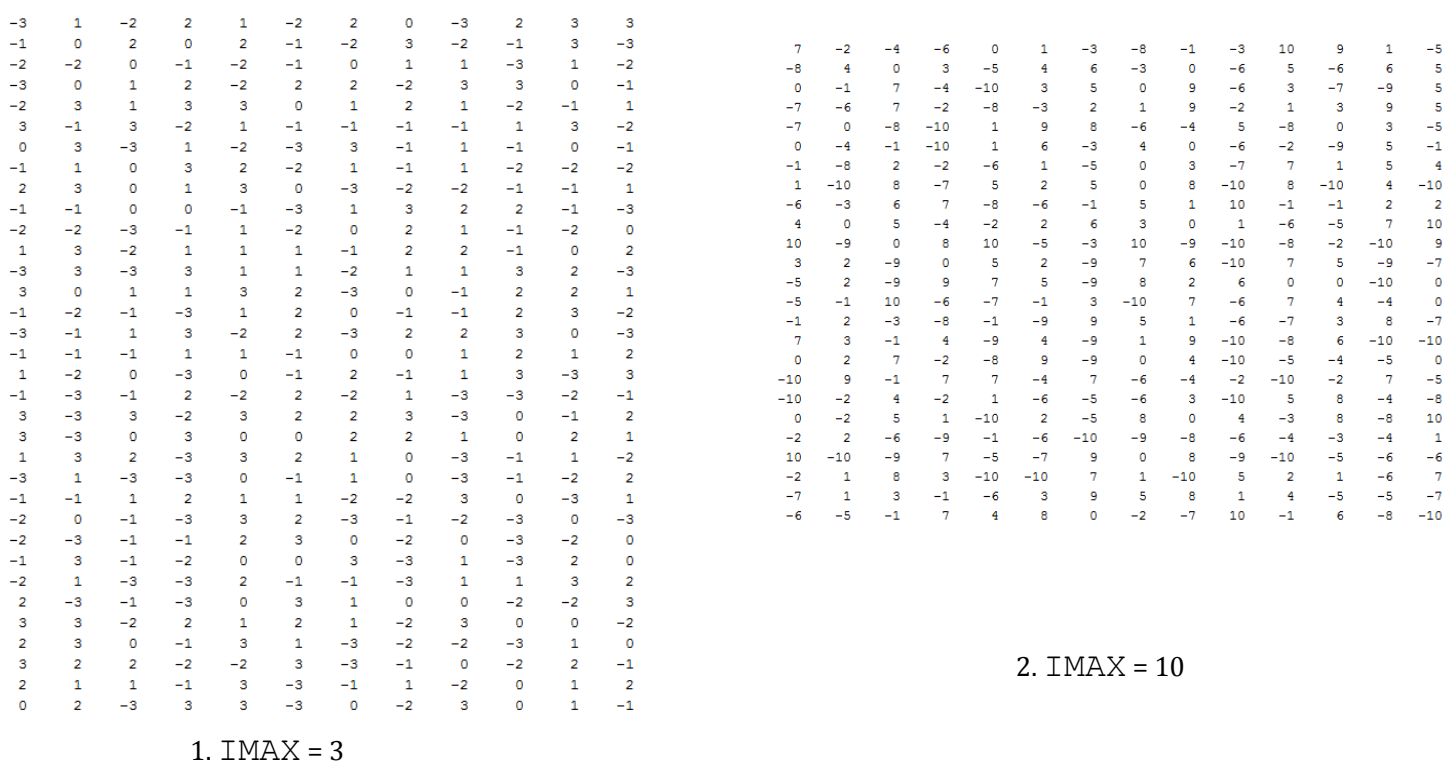

Figure 16: The image matrices with random noise. 


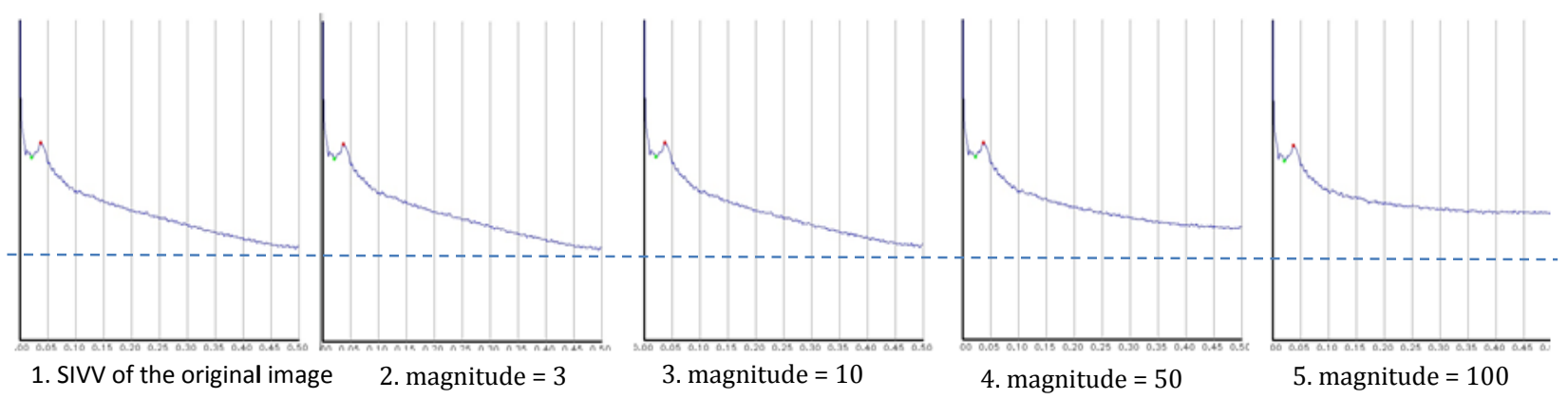

Figure 17: The comparison of SIVV curves of an image with different levels of IID random noise.

\section{EXPERIMENTAL RESULTS}

\subsection{LATENT FINGERPRINT PREPROCESSING DATASET}

As a preliminary means to demonstrate objective assessment of latent fingerprint enhancement, we compared the SIVV characteristics of fingerprint images pre- and postenhancement. A training dataset was provided for our study. In the dataset, there are six types of latent fingerprint images: Bi-Chromatic mag powder developed prints, Bi-Chromatic powder developed prints, black ink pad on colored background, Ninhydrin developed prints, silver mag powder developed prints, and white powder developed prints as shown in Figure 18. We use 39 forensic latent fingerprint image pairs in our experiment. The number of pairs in the six types is not balanced. For each image pair, the before image is an RGB color image which was scanned by a high-resolution flatbed scanner. A Certified Latent Print Examiner (CLPE) preprocessed the before image within Adobe Photoshop (which is the primary image analysis tool used by CLPE's practicing today), converted it to grey image, and saved it as tiff format as the after image. The before and after image pairs are both in the same size, resolution, and saved in tiff format. Each image contains at least one latent fingerprint. The background for some images is very noisy. The fingerprint ridges and furrows are in low contrast and very blurry. Some images only contain a partial fingerprint image (less than $1 / 4$ fingerprint). 


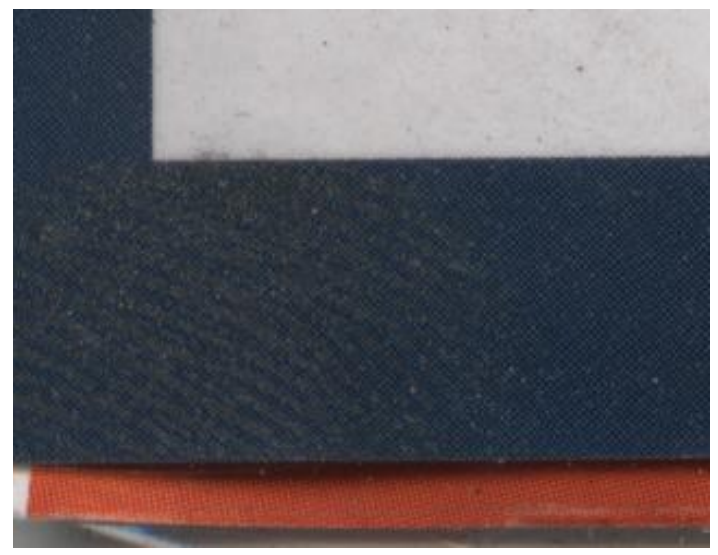

(1) Bi-Chromatic Mag Powder Developed Prints

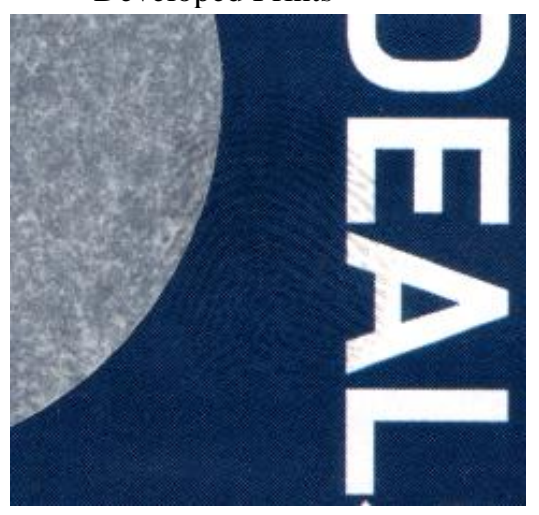

(3) Black ink pad on colored background

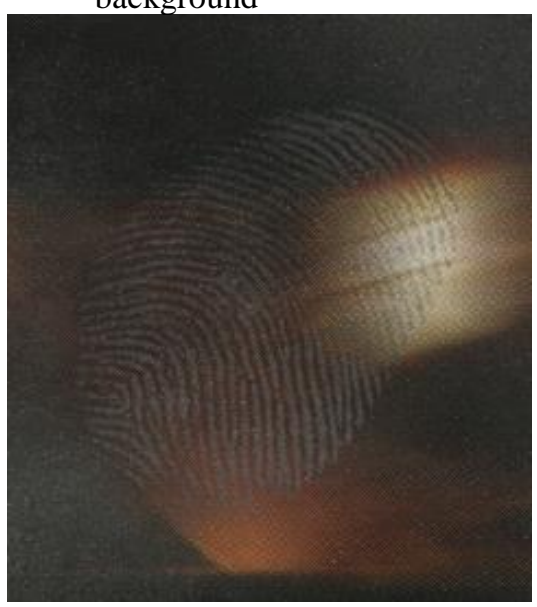

(5) Silver mag powder developed prints

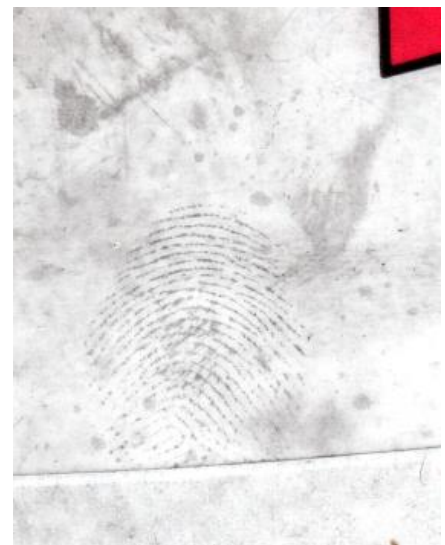

(2) Bi-Chromatic Powder Developed Prints

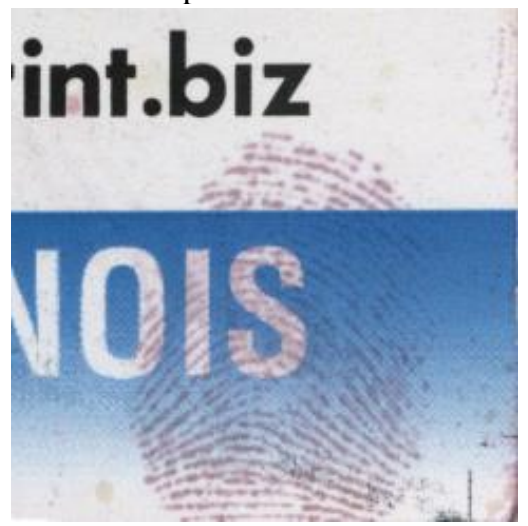

(4) Ninhydrin developed prints

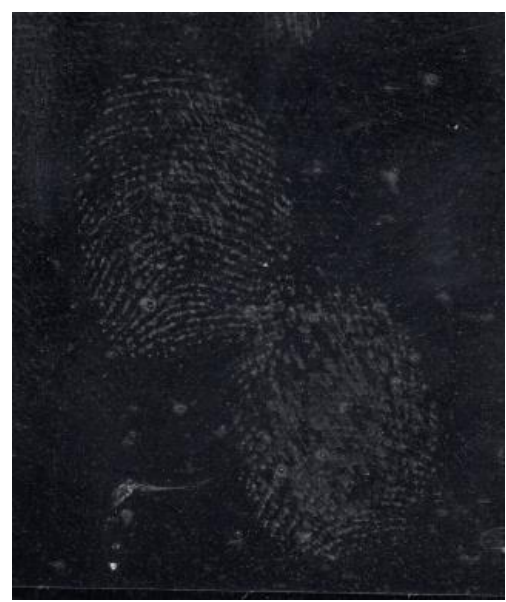

(6) White powder developed prints

Figure 18: The latent fingerprint preprocessing image pairs from a training course (six types). 


\subsection{LATENT FINGERPRINT QUALITY MEASUREMENT}

Our SIVV algorithm including modifications for latent fingerprint image quality measurement is diagrammed in Figure 19. The blue color represents original implementation. The green color represents new components. In the proposed algorithm implementation, the option of a manual ROI selection GUI interface is added, so that the user will interactively select the region of the fingerprint. The new algorithm also revised the peak location detection module in order to select the correct peak using a peak location constraint.

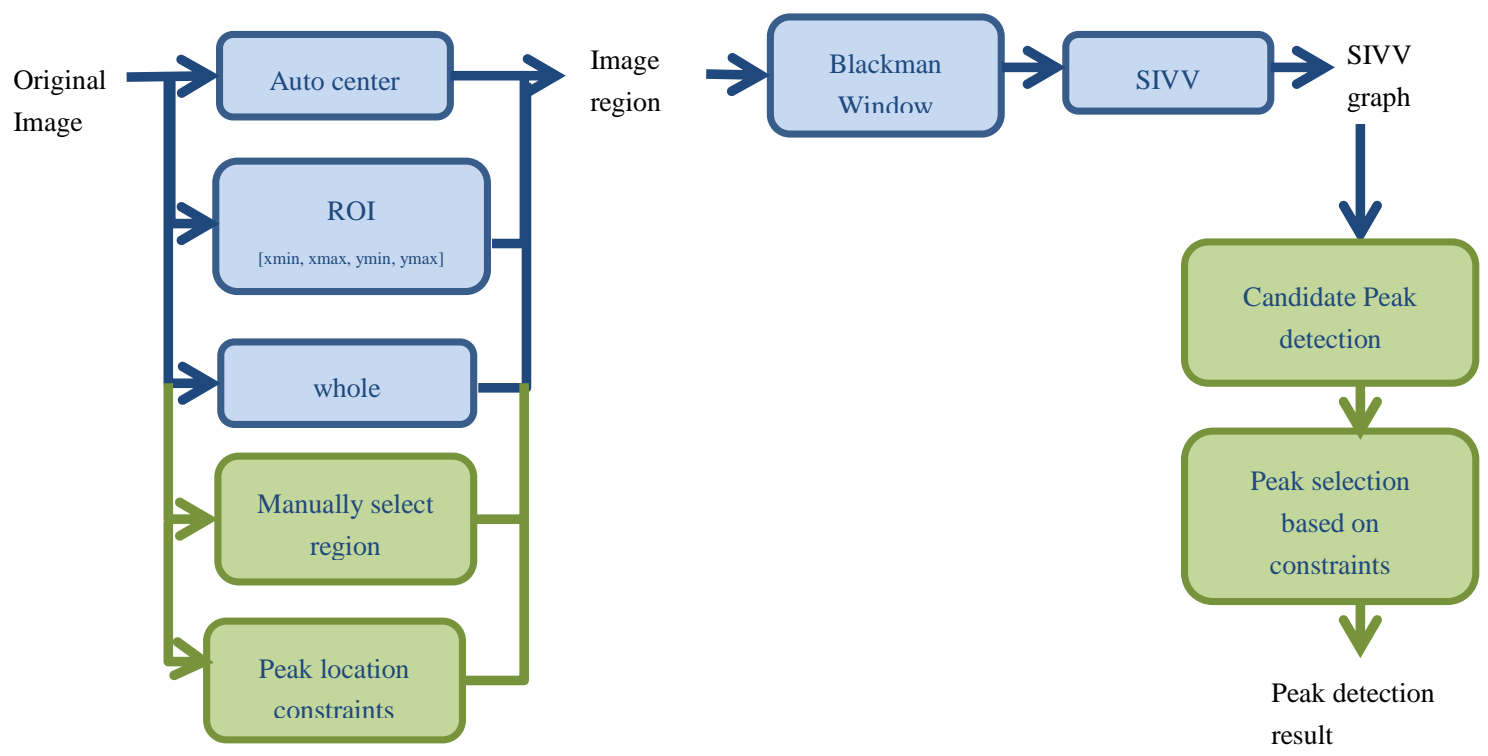

Figure 19: The proposed algorithm for latent fingerprint quality measurement.

\subsubsection{Region of interest}

For some images in our dataset (Figure 7, cases 2 and 4), the background noise also includes a strong repeated texture pattern, which provides a strong noise peak in the SIVV curve. Instead of representing the fingerprint ridge information, this peak represents the background texture pattern. In order to reduce the background noise, we need to select the fingerprint region and calculate the SIVV curve only on ROI image, which contains strong fingerprint level-one features instead of background noise. 


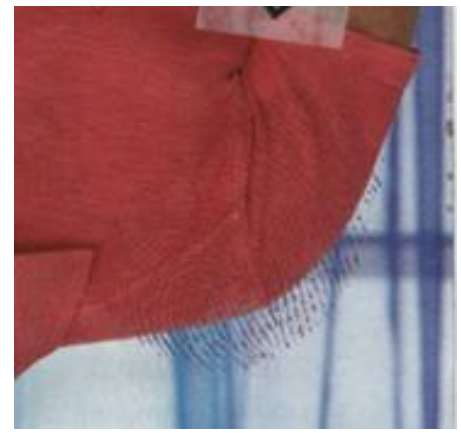

1. Original image

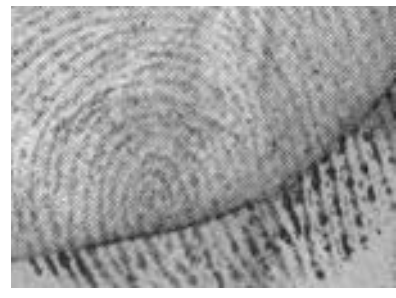

2. ROI

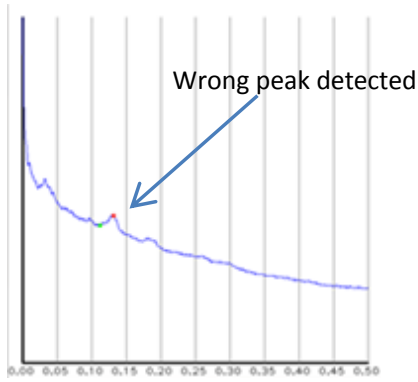

2. SIVV of original image

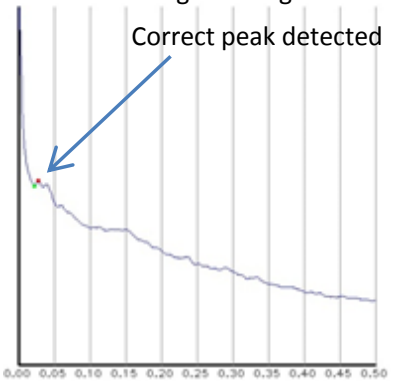

4. SIVV of ROI

Figure 20: The comparison of whole image vs. ROI image.

Due to the poor quality of the latent fingerprints, automatic ROI extraction is a very challenging problem. In the present experiment, we implemented a graphical user interface to manually select rectangular ROI on the displayed input image. In the future, we may explore the use of a polygon to enhance the accuracy. In addition, we may also propose a semiautomatic ROI extraction method where, given the center of the fingerprint region and the radius of an ellipse which roughly covers the ROI, the algorithm automatically finds the maximum of the strongest SIVV signal peak and locates the accurate boundary of the ellipse. Figure 20 shows that when the SIVV algorithm takes the whole image as input, it detects the wrong peak which actually represents the texture noise, while if we use a manually selected ROI as input for the analysis, the algorithm correctly detects the fingerprint peak.

\subsubsection{Peak location constraint}

Within the ROI, we implemented an additional interface that allows the user to draw line segments from one ridge perpendicular to itself to another adjacent ridge. The user can draw multiple segments including some in both dense and sparse ridge areas. These fiducial lengths serve as benchmark estimates of the ridge-to-ridge distance measured in pixels which, in turn, helps to anchor the spectral search region for the SIVV analysis. As long as the background noise's spectrum is smooth throughout the frequencies containing fingerprint ridge information, the SIVV algorithm will identify the corresponding peak range in the specified and limited frequency domain. 


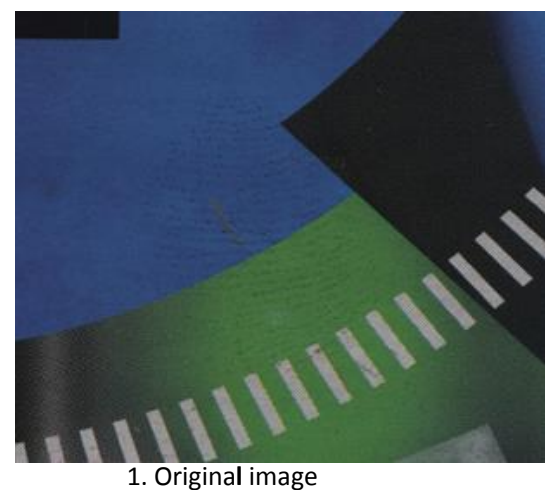

1. Original image

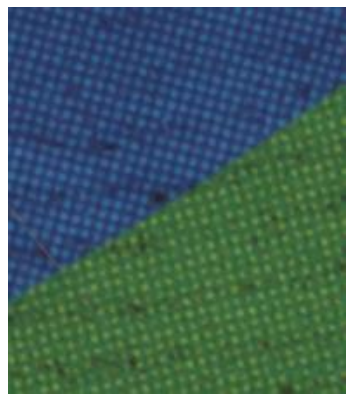

3. Zoom in to see the background noise texture

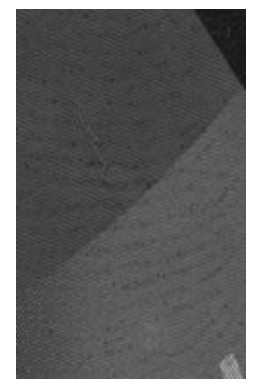

2. ROI

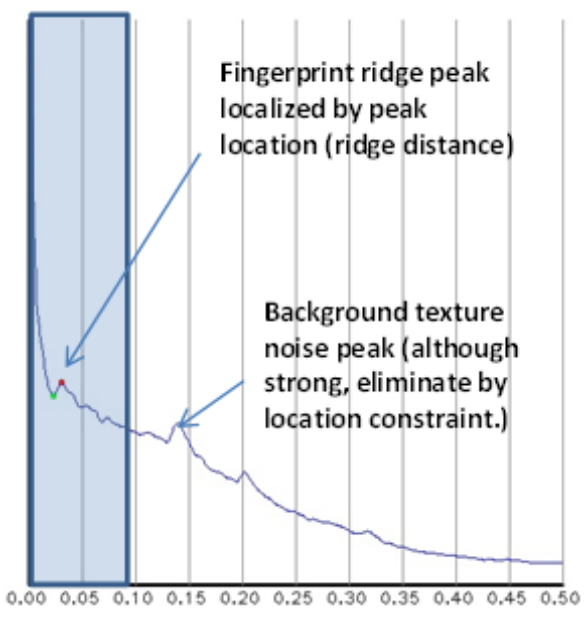

4. SIVV peak (fingerprint)

Figure 21: Peak location constraint.

Figure 21 shows an example of using a location constraint to find the correct fingerprint peak. The strongest peak is around the location at 0.15 cycles per pixel. Zooming in on the image in Figure 21-3 reveals a pervasive uniform grid texture in the image background. From elementary analysis, we may conclude that the strong peak around 0.15 is actually not the fingerprint peak, but rather represents the frequency of the background texture. According to multiple estimates of the fingerprint ridge distance in the image, the possible spatial frequency range for the fingerprint SIVV peak is indicated by the blue bar. The actual SIVV peak is the weak peak in the blue bar around 0.03 cycles per pixel. The image analyzed in Figure 21 is an example "before image" from our dataset. Figure 22 shows the image obtained after preprocessing. The combination of ROI selection and marking interridge distances diminishes the spectral peak due to the background texture and allows for automatic detection of the SIVV feature in the polar spectrum plot. 


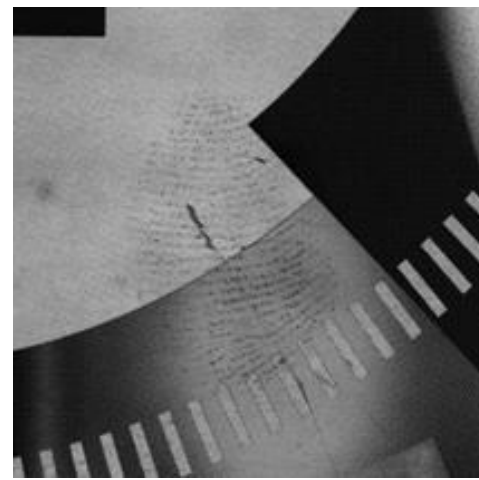

1. Original image (037 enhanced)

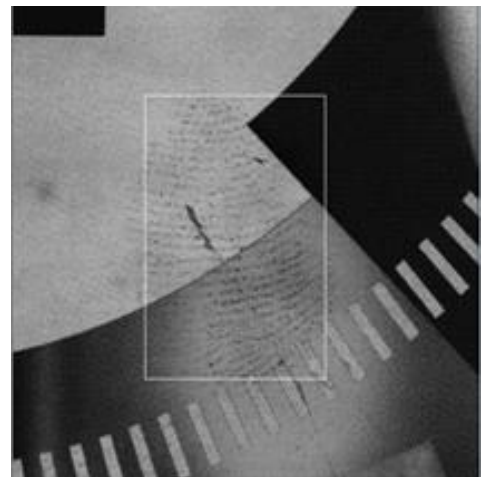

2. Selected ROI

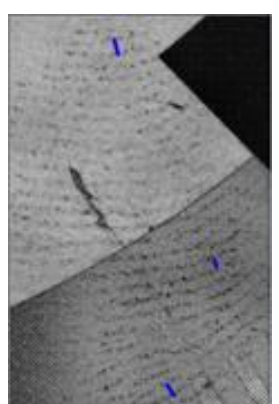

3. Specify ridge distances ( $\max , \min )$

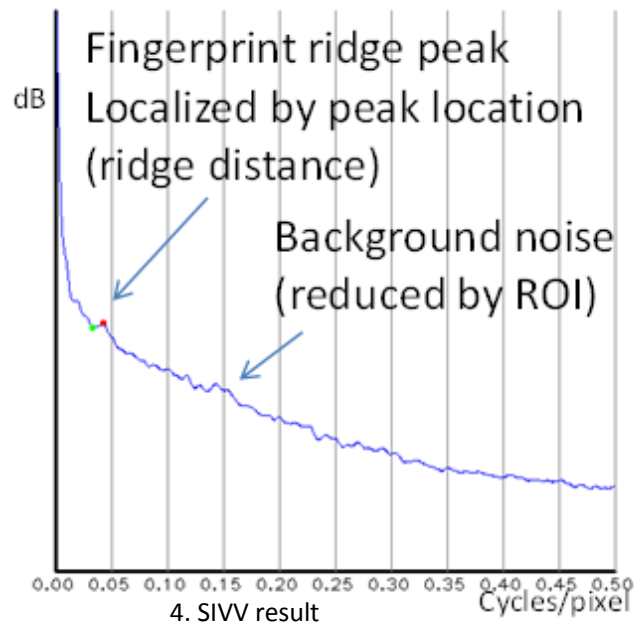

Figure 22: Proposed procedure for latent fingerprint SIVV feature detection.

Figure 23 shows the correct peaks are detected using peak location constraints in different latent fingerprint images. 

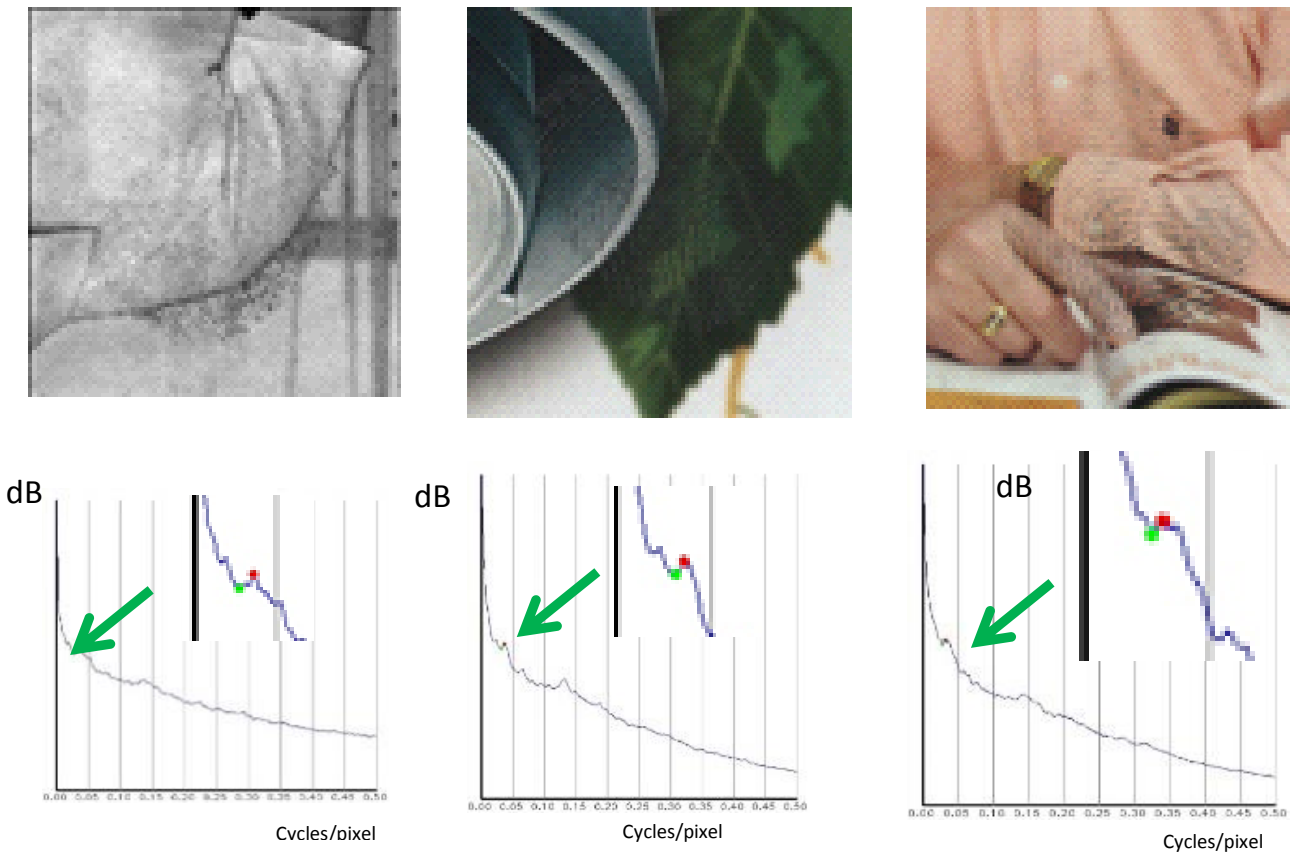

Figure 23: The frequency feature of latent fingerprint using the proposed algorithm.

\subsection{COMPARISON RESULTS}

We performed five experiments on our latent fingerprint preprocessing dataset to demonstrate the efficacy of the SIVV modifications described above in concert with latent fingerprint image preprocessing. If the correct SIVV peak is detected, we count it as true positive. We calculate the true positive rate (TPR): TPR = True Positive (TP) / (True Positive + False Negative). The five experiments are:

(a) The SIVV implementation provided by the NBIS software package [35], using the default option (all parameters are set to default values). In this setting, the algorithm cuts the image first, then applies the Blackman filter, and calculates SIVV curve.

(b) The SIVV implementation provided by NBIS, using the whole image option (all other parameters are set to default values).

(c) The SIVV implementation provided by NBIS, with a resized input image (half of original width and height) (all other parameter are set to default values). In this setting, the algorithm cuts the image first (different region from experiment a), then applies the Blackman filter, and calculates SIVV curve.

(d) A modified SIVV analysis including manual selection of ROI using a GUI interaction (all other parameters are set to default values).

(e) A modified SIVV analysis including both manual selection of ROI and manual input of the ridge distances by line segments using a GUI (all other parameters are set to default values). 
The results are shown in Table 1. Clearly the successful detection rates of the preprocessed images (the values in the second row) are consistently higher than the unprocessed counterparts (the values in the first row). Based on this, we conclude that preprocessing can be useful in amplifying fingerprint information contained in latent fingerprint images.

Table 1: The comparison of different algorithms with different options

\begin{tabular}{|c|c|c|c|c|c|}
\hline TPR = TP/(TP+FN) & $\begin{array}{c}\text { Original } \\
\text { image } \\
\text { Default } \\
\text { option }\end{array}$ & $\begin{array}{c}\text { Original } \\
\text { image } \\
\text { Whole option }\end{array}$ & $\begin{array}{c}\text { Resize image } \\
\text { Default } \\
\text { option }\end{array}$ & $\begin{array}{c}\text { Original } \\
\text { image } \\
\text { GUI ROI }\end{array}$ & $\begin{array}{c}\text { GUI ROI } \\
\text { Peak loc. } \\
\text { Constraint }\end{array}$ \\
\hline Before & $36 \%$ & $33 \%$ & $62 \%$ & $79 \%$ & $85 \%$ \\
\hline After & $64 \%$ & $72 \%$ & $82 \%$ & $87 \%$ & $92 \%$ \\
\hline
\end{tabular}

\section{DISCUSSION AND FUTURE WORK}

The Spectral Image Validation/Verification (SIVV) analysis was introduced to screen fingerprint image databases for low-quality and/or non-fingerprint images. It was observed in that, "The magnitude of the distinctive spectral feature, related directly to the distinctness of the level 1 ridge flow, provides a primary diagnostic indicator of the presence of a fingerprint image," [1]. While effective for controlled capture fingerprints, the diagnostic capability of SIVV is significantly impaired in the context of low-quality latent fingerprint images. We have introduced modifications to SIVV to restore this capability. Furthermore, we demonstrate that the modified SIVV analysis can be used as a quality indicator for preprocessing, and that it is an essential precursor to detailed forensic investigation of latent fingerprint images. 


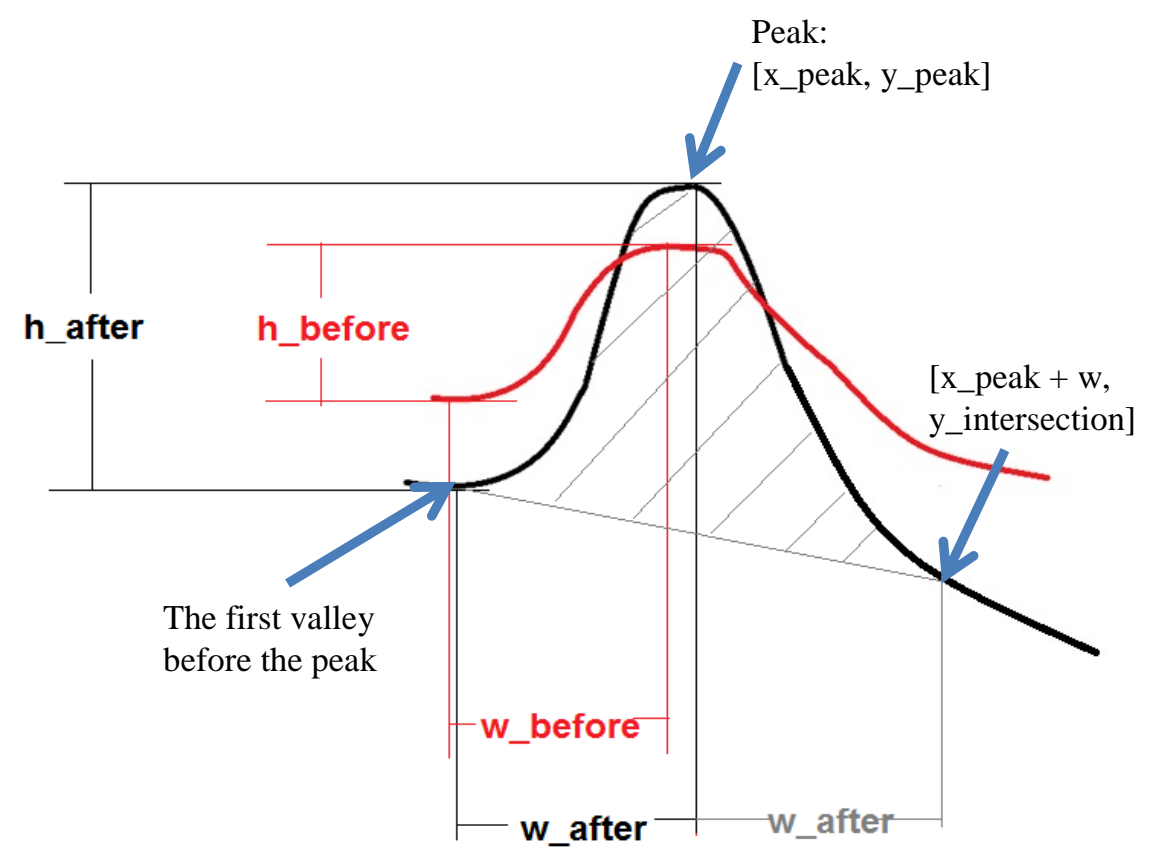

Figure 24: Quantitative comparison of latent fingerprint image quality.

The systematic investigation of forensic latent fingerprint preprocessing is still in an early stage and much work remains to be done so as to place this critical component of forensic analysis on firmer quantitative foundations. The current SIVV analysis inspects the log polar power spectrum for a characteristic peak-trough feature indicative of the ridge periodicity (averaged over angles) exhibited by a fingerprint. The possibility exists that this spectral feature could be further refined by computing peak-height and/or area between two SIVV curves after suitable normalization and alignment. For example, if we define the peak height, $\mathrm{h}$, as the height between the two points: the peak (the local highest point) and the first valley before the peak (the left local lowest point), the relative differences of the peak height is $\Delta \mathrm{h}$ $=h_{\text {after- }} h_{\text {before, }}$ as shown in Figure 24 . If we define the peak width, $w$, as the horizontal distance between those two points, the relative differences of the peak width is $\Delta \mathrm{w}=\mathrm{w}_{\text {after- }}$ Wbefore, as shown in Figure 24 (which is approximately the half of the total hump width). Because the end point of the hump is very ambiguous, we define it to be the intersection of the curve and the vertical line which is the same w distance from the peak on the right. We can also define the peak area and its difference, $\Delta s=s_{\text {after }}$-Sbefore. In doing this, a finer quantitative understanding of the quality of fingerprint information contained in an image might be developed. We hope to report on such investigations in the future. 


\section{ACKNOWLEDGEMENTS}

The authors thank John M. Libert, John Grantham, and Shahram Orandi of NIST for their valuable contributions to this work. The authors thank Mathew Schwarz of Schwarz Forensics and David Witzke of Foray Technologies for valuable consultation on this work. This research was supported by the 2012 NIST Forensic Measurement Challenges grant, "Metrics for Manipulation and Enhancement of Forensic Images."

\section{DISCLAIMER}

Any mention of commercial products or reference to commercial organizations in this report is for information only; it does not imply recommendation or endorsement by NIST nor does it imply that the products mentioned are necessarily the best available for the purpose.

\section{REFERENCES}

[1] John M. Libert, John Grantham, and Shahram Orandi, "A 1D spectral image validation/verification metric for fingerprints," NISTIR 7599, 2009.

[2] Edward M. Robinson, second edition, Crime scene photography. Chapter 10, Digital Imaging Technologies, contributions by David "Ski” Witzke, Academic Press, 2010.

[3] Andrew M. Dienstfrey, et al., "Analysis of Image Enhancement Operations Applied to Latent Fingerprints," NIST IR, Gaithersburg, MD, 2014.

[4] Peter Bajcsy, Joe Chalfoun, David A. Nimorwicz, and Andrew M. Dienstfrey, "Estimation of Mathematical Models Relating Latent Fingerprint Images Before and After Enhancement," NIST IR, Gaithersburg, MD, 2013.

[5] Mary Theofanos, Brian Stanton, et al., "Characterizing the Latent Fingerprint Preprocessing Procedures,” NIST IR, Gaithersburg, MD, 2014.

[6] David Nimorwicz, Joe Chalfoun, and Peter Bajcsy, "Comparison Metrics for Latent Fingerprint Images Before and After Enhancement," NIST IR, Gaithersburg, MD, 2013 
[7] Alfred S. Carasso, Alternative Methods of Latent Fingerprint Enhancement and Metrics for Comparing Them, NISTIR 7910, 2013.

[8] Alfred S. Carasso, "The use of 'slow motion' Levy Stable Fractional Diffusion Smoothing in Alternative Methods of Latent Fingerprint Enhancement," NISTIR 7932, 2013.

[9] NIST Special Database 27, "Fingerprint Minutiae from Latent and Matching Tenprint Images," http://www.nist.gov/srd/nistsd27.cfm.

[10] Michael D. Indovina, V. N. Dvornychenko, E. Tabasse, G. Quinn, P. Grother, M. Garris, and S. Meagher, "ELFT Phase II: An Evaluation of Automated Latent Fingerprint Identification Technologies," U.S. Department of Commerce, National Institute of Standards and Technology, 2009.

[11] Robert Yen and Joseph Guzman. "Fingerprint image quality measurement algorithm," Journal of Forensic Identification 57, no. 2, pp. 274, 2007.

[12] Julian Fierrez-Aguilar, Yi Chen, Javier Ortega-Garcia, and Anil Jain, "Incorporating image quality in multi-algorithm fingerprint verification," Advances in Biometrics, pp 213-220, 2005.

[13] Soweon Yoon, Jianjiang Feng, and Anil K. Jain. "On latent fingerprint enhancement," SPIE Defense, Security, and Sensing, International Society for Optics and Photonics, 2010.

[14] Soweon Yoon, Eryun Liu, and Anil K. Jain. "On latent fingerprint image quality,” In Proc. International Workshop on Computational Forensics. 2012.

[15] Soweon Yoon, Kai Cao, Eryun Liu, and Anil K. Jain, "LFIQ: Latent fingerprint image quality," 2013 IEEE Sixth International Conference on Biometrics: Theory, Applications and Systems (BTAS), pp. 1-8. IEEE, 2013.

[16] M. A. U. Khan, "Fourier cleaning of fingerprint images," 2010 IEEE 10th International Conference on Signal Processing (ICSP), pp. 2604-2607. IEEE, 2010.

[17] B. G. Sherlock, D. M. Monro, and K. Millard, "Fingerprint enhancement by directional Fourier filtering," IEE Proceedings Vision, Image and Signal Processing, vol. 141, no. 2, pp. 87-94. IET, 1994. 
[18] R.T Moore, "An analysis of ridge-to-ridge distance on fingerprints," J. Forensic Identification, vol. 39, pp. 231-238, 1989.

[19] Michael D. Garris, and R. Michael McCabe, "NIST Special Database 27: Fingerprint minutiae from latent and matching tenprint images," NIST, Gaithersburg, MD. [CDROM] NISTIR 6534.

[20] NIST Biometric Image Software, http://www.nist.gov/itl/iad/ig/nbis.cfm, NIST USA.

[21] Michael W. Reidling, "Recording and Using Photoshop Actions to Streamline Workflow in Latent Enhancement and Crime Scene Photography," Presented at the International Association for Identification's 97th International Educational Conference on 24 July 2012. http://onin.com/fp/.

[22] Scott Hecker Gische, Glenn Langenburg, and Alice Maceo, "SWGFAST Response to The Research, Development, Testing \& Evaluation Inter-Agency Working Group of the National Science and Technology Council, Committee on Science, Subcommittee on Forensic Science," 2011.

[23] H. Edwards, and C. Gotsonis, "Strengthening forensic science in the United States: a path forward," Statement before the United State Senate Committee on the Judiciary. Chapter 10, Automated Fingerprint Identification Systems, 2009.

[24] John C. Russ, "Forensic uses of digital imaging," CRC, pp. 549, 2001.

[25] Bramble, Simon, David Compton, and L. Klasén, "Forensic image analysis," In Proc. of 13th INTERPOL Forensic Science Symposium, Lyon FR. 2001.

[26] A. Hicklin, and C. Reedy, "Implications of the IDENT/IAFIS Image Quality Study for Visa Fingerprint Processing," Mitertek Systems (MTS), 2002.

[27] Tai Pang Chen, Xudong Jiang, and Wei Yun Yau, "Fingerprint image quality analysis," 2004 International Conference on Image Processing (ICIP'04). Vol. 2. IEEE, 2004.

[28] Yi Chen, Sarat Dass, and Anil Jain, "Fingerprint quality indices for predicting authentication performance," In Audio-and Video-Based Biometric Person Authentication, pp. 160-170. Springer Berlin/Heidelberg, 2005. 
[29] Elham Tabassi, Charles L. Wilson, and Craig I. Watson. Fingerprint image quality. U.S. Department of Commerce, National Institute of Standards and Technology, 2004.

[30] Jacqueline A. Speir, Jack Hietpas, Tara Fikes, "Fast Fourier transformation and frequency filtering to suppress background noise in fingerprint evidence: quantifying the fidelity of digitally enhanced fingerprint evidence," Cedar Crest College, Allentown PA, Syracuse Univ., Syracuse, NY.

[31] Bradford T. Ulery, R. Austin Hicklin, JoAnn Buscaglia, and Maria Antonia Roberts, "Accuracy and reliability of forensic latent fingerprint decisions," Proceedings of the National Academy of Sciences 108, no. 19, pp. 7733-7738, 2011.

[32] Sargur N. Srihari and Graham Leedham, "A survey of computer methods in forensic document examination," In Proceedings of the 11th Conference of the International Graphonomics Society, pp. 279. 2003.

[33] Matthew Stamm and KJ Ray Liu, "Blind forensics of contrast enhancement in digital images," 15th IEEE International Conference on Image Processing (ICIP'08), pp. 31123115, IEEE, 2008.

[34] Foray, "calibrating your images," http://www.foray.com/images/pdfs/CalibratingYourImages.pdf.

[35] Flynn Mcroberts and Steve Mills, Chicago Tribune, "FBI's digital fingerprints could collar the innocent," http://articles.dailypress.com/2005-01-

04/news/0501040066_1_inked-print-cards-prominent-fingerprint-latent-prints-trainingcoordinator, January 04, 2005.

[36] Chicago Tribune, "Digital fingerprint technology can point to wrong person," The Baltimore Sun, http://articles.baltimoresun.com/2005-0130/news/0501290073_1_digital-image-digital-fingerprint-computer-images, January 30, 2005.

[37] Photo Finish, online document, www.fdiai.org/articles/Photo\%20Finish.pdf. 


\section{APPENDIX A: THE SIVV PEAK ON SYNTHETIC IMAGES}

In order to study the properties of SIVV and find out the relationship between the SIVV peak location and texture frequencies, we generated a set of synthetic images. The first image set is a set of black/white stripe images with different stripe width. The size of the images in the first image set is 512 by 512. For each image, given the width of the stripes, we generate the image with black/white stripes with equal width. Figure 25 shows the comparisons of SIVV peaks of images with the different stripe width. Table 2 shows the stripe width and their SIVV peak location. In the frequency range of the texture pattern that we consider, ${ }^{5}$ the peak location follows a certain rule. Generally, the narrow stripe image's SIVV peak location is smaller (or closer to the origin) than the wide stripe image's peak location for the same texture pattern.

The size each image in the second image set is 1024 by 1024 in Figure 26. Similarly as the experiment on the first image set, given the width of the stripes, the images are synthesized and their frequency feature are extracted. Table 3 shows the stripe width and their SIVV peak locations. Comparing Table 2 and Table 3, firstly, it indicates that the peak location is not related to the image size. It is reasonable because the $\mathrm{x}$-axis of the SIVV curve is the cycles per pixel. The $\mathrm{x}$-axis is normalized by the image size in the last step of SIVV algorithm. Secondly, the SIVV peak location is related with the width of the stripe directly. For example, the peak location of the image with four-pixel stripe width is 0.25 for both image with the size of 512 by 512 and the image with the size of 1024 by 1024 . The peak location is 0.125 of the images with eight-pixel stripe width for both image sizes. For the same texture pattern (as our stripe pattern example), the peak location is decided by the pixel distance of the pattern width. The peak location follows the same rule as the first set: the SIVV peak location of the image with the narrow stripe is closer to the origin than the one of the image with wide stripe.

On the other hand, notice that the image B in Set 2 (resolution: 1024×1024; stripe width: 8 pixels) is just the high-resolution version of the image A in Set 1 (resolution: 512×512; stripe width: 4 pixels). If we resample image $B$ in Set 2 by taking every other pixel in both column and row, we obtain the image A in Set 1. PPI (Pixels Per Inch) of the first image (the image $\mathrm{B}$ in Set 2) is twice as the second image (the image A in Set 1). In our case, if we scan the same forensic latent fingerprint with different PPI, the peak location of high PPI (B in set 2)

\footnotetext{
5 We study the texture patterns in a certain frequency range. If the frequency is too high (i.e., the stripe width is 1 or 2 pixels for the image with size $512 \times 512$, SIVV curve does not show peaks. If the frequency is too low, SIVV shows peaks in multiple locations (like a wave). In our study, we consider only the frequency in a certain range, which is relatively high compared with the image size. No extreme case is considered here.
} 
is half of the peak location of low PPI image (A in Set 1). Table 4 shows the comparison results.

In summary, the SIVV peak location is directly related to the stripe width in pixel distance. Table 5 shows the peak location given the stripe width. We may use it as a reference for the proposed peak location constraint algorithm.

Finally, we also generate another set of synthetic image with square pattern (as shown in Figure 27). It shows that SIVV peak location follows the same rule; just the location of the peak is different given different texture pattern. 


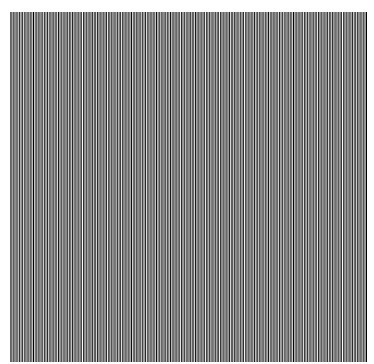

Set 1 A-1. width: $4(512 \times 512)$

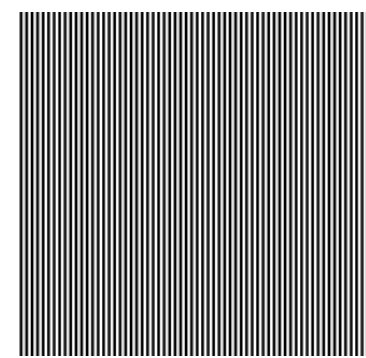

Set 1 B-1. width: $8(512 \times 512)$

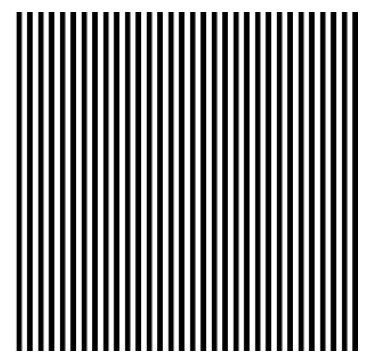

Set 1 C-1. width: $16(512 \times 512)$

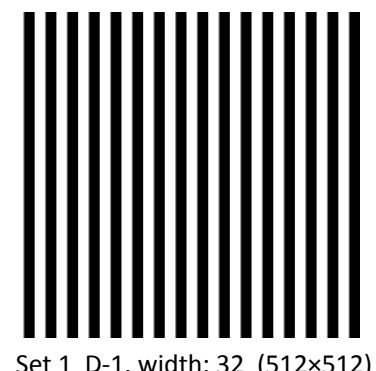

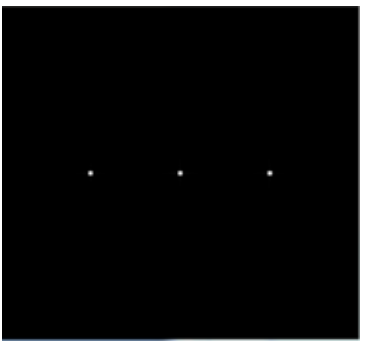
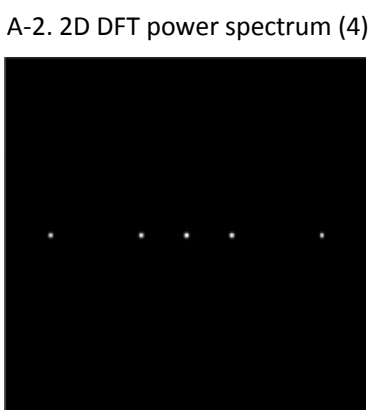

B-2. 2D DFT power spectrum (8)

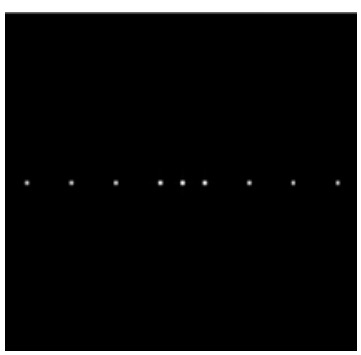

C-2. 2D DFT power spectrum (16)

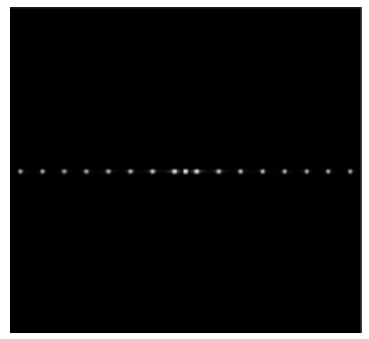

D-2. 2D DFT power spectrum (32)

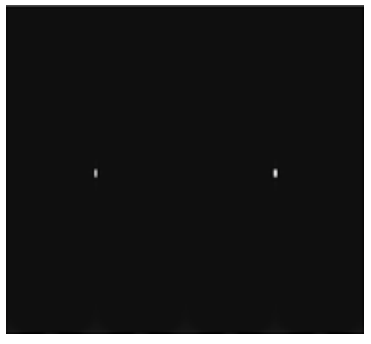

A-3. 2D Polar spectrum (4)

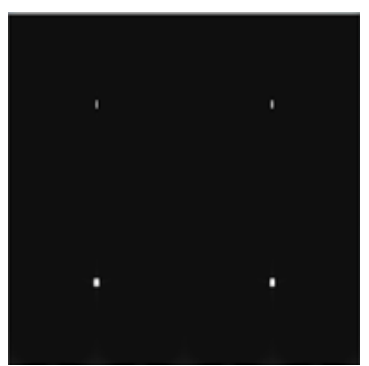

B-3. 2D Polar spectrum (8)

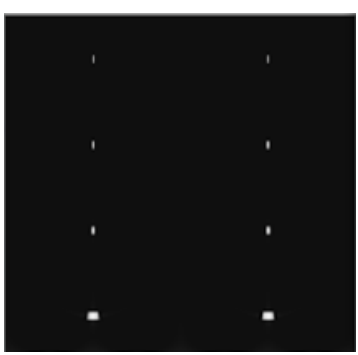

C-3. 2D Polar spectrum (16)

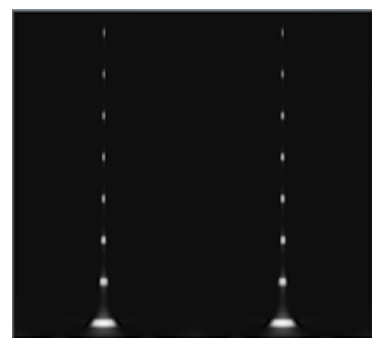

D-3. 2D Polar spectrum (32)
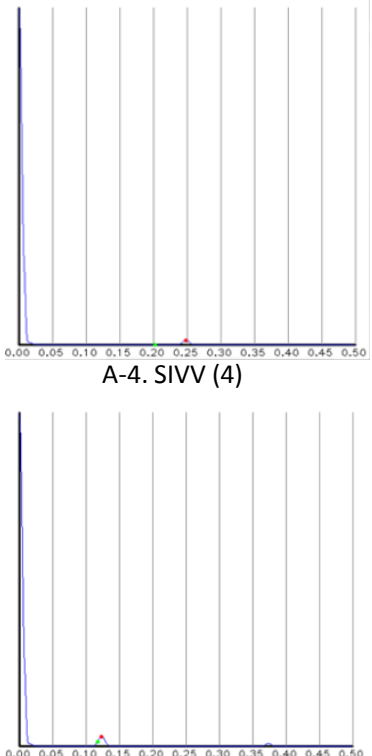

B-4. SIVV (8)

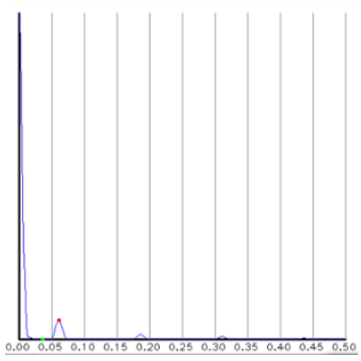

C-4. SIVV (16)

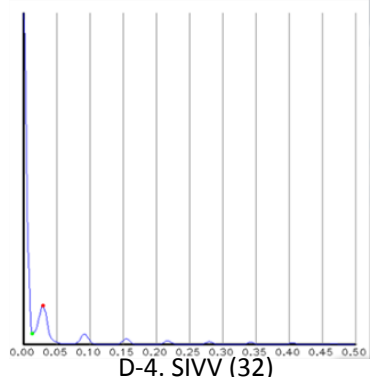

Figure 25: SIVV on the images with the stripes pattern (image size $512 \times 512$ pixels). 


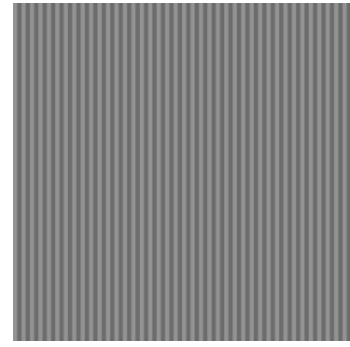

Set 2 A-1. width: $4(1024 \times 1024)$ A-2. 2D DFT power spectrum (4)

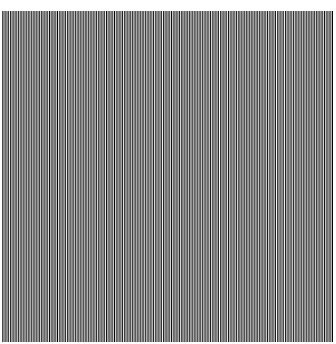

Set 2 B-1. width: $8(1024 \times 1024)$

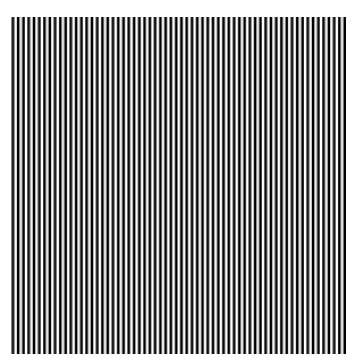

Set 2 C-1. width: $16(1024 \times 1024)$ C-2. 2D DFT power spectrum (16)

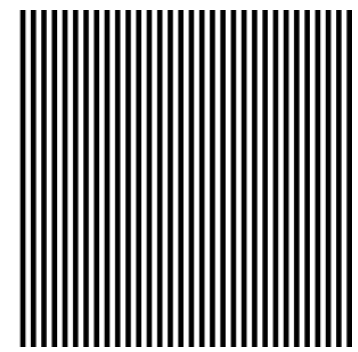

Set 2 D-1. width: $32(1024 \times 1024)$ D-2. 2D DFT power spectrum (32)
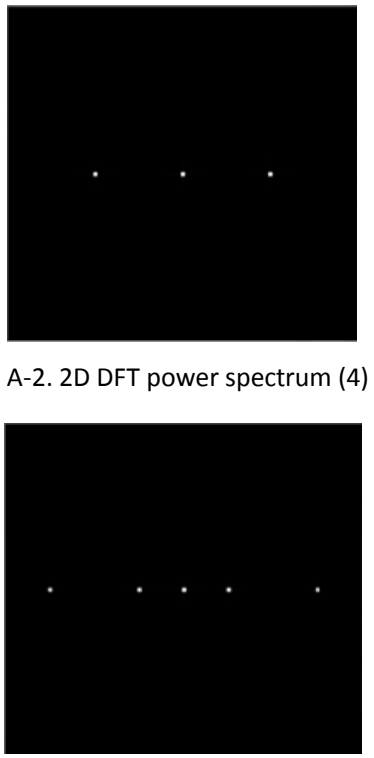

B-2. 2D DFT power spectrum (8)
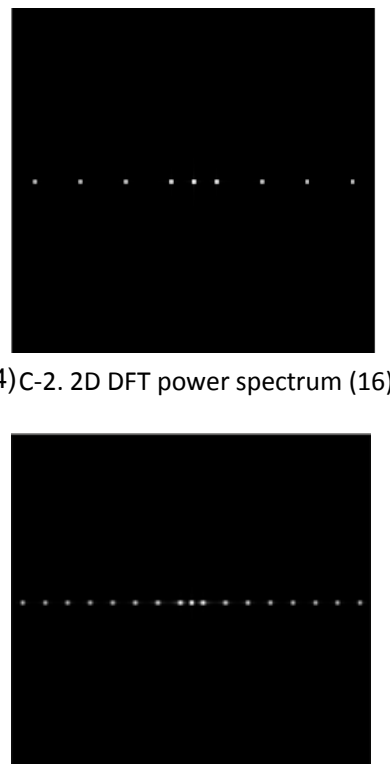

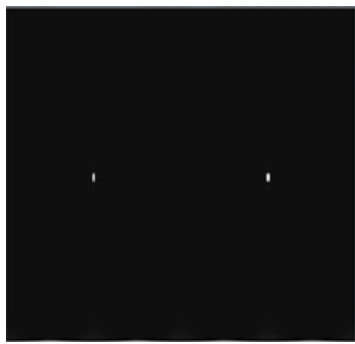

A-3. 2D Polar spectrum (4)

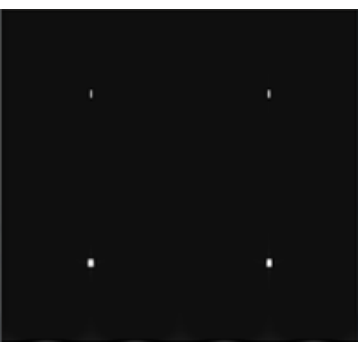

B-3. 2D Polar spectrum (8)

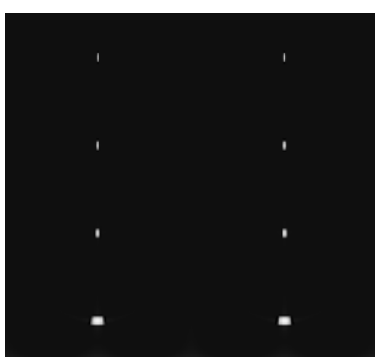

C-3. 2D Polar spectrum (16)

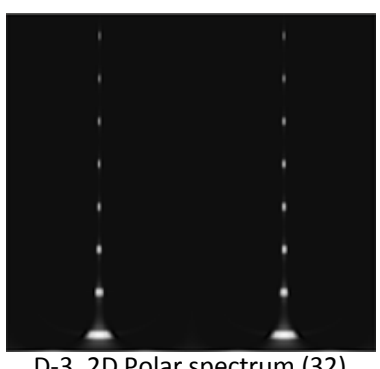

D-3. 2D Polar spectrum (32)

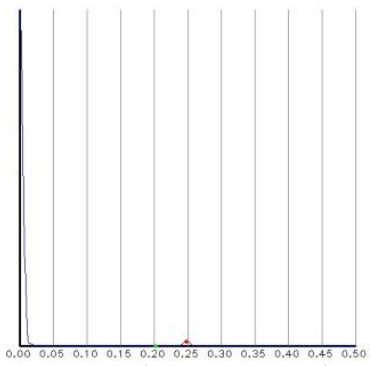

A-4. SIVV (4; location 0.25 )

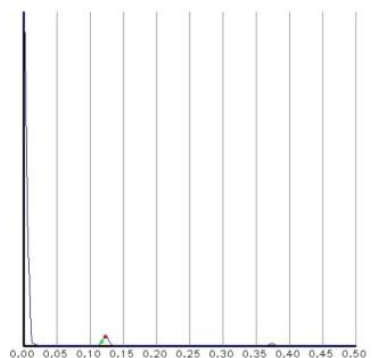

B-4. SIVV (8; location 0.125)

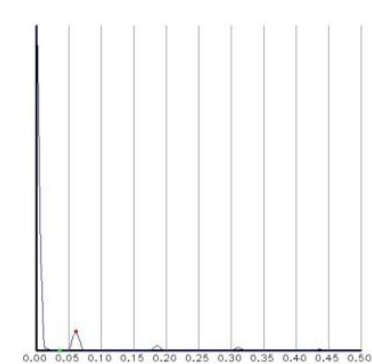

C-4. SIVV (16; location 0.0625)

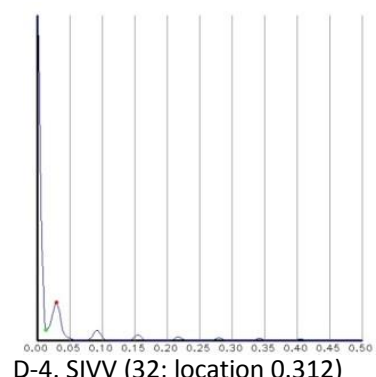

Figure 26: SIVV on the large images with the stripes pattern (image size $1024 \times 1024$ pixels).

Table 2: SIVV peak locations on the images with the stripes pattern (image size $512 \times 512$ pixels)

\begin{tabular}{|l|l|l|l|l|}
\hline Stripe width & 4 & 8 & 16 & 32 \\
\hline
\end{tabular}




\begin{tabular}{|l|l|l|l|l|}
\hline Peak location & 0.25 & 0.125 & 0.625 & 0.312 \\
\hline
\end{tabular}

Table 3: SIVV peak locations on the large images with the stripes pattern (image size $1024 \times 1024$ pixels)

\begin{tabular}{|l|l|l|l|l|}
\hline Stripe width & 4 & 8 & 16 & 32 \\
\hline Peak location & 0.25 & 0.125 & 0.625 & 0.312 \\
\hline
\end{tabular}

Table 4: The relationship between peak locations and PPI

\begin{tabular}{|c|c|c|c|}
\hline $512 \times 512$ Strip width & 4 & 8 & 16 \\
\hline $512 \times 512$ Peak location & 0.25 & 0.125 & 0.625 \\
\hline $\begin{array}{l}\text { Double PPI of first row } \\
\text { (the image looks the } \\
\text { same, but the resolution } \\
\text { is doubled in both } x \text {, and } \\
\text { y directions) }\end{array}$ & & & \\
\hline $1024 \times 1024$ strip width & 8 & 16 & 32 \\
\hline $1024 \times 1024$ peak location & 0.125 & 0.0625 & 0.0312 \\
\hline
\end{tabular}

Table 5: The peak locations given the stripe pixel distance.

\begin{tabular}{|l|l|l|l|l|l|l|l|l|l|}
\hline $\mathbf{4}$ & $\mathbf{8}$ & $\mathbf{1 2}$ & $\mathbf{1 6}$ & $\mathbf{2 0}$ & $\mathbf{2 4}$ & $\mathbf{2 8}$ & $\mathbf{3 2}$ & $\mathbf{3 6}$ & $\mathbf{4 0}$ \\
\hline 0.25 & 0.125 & 0.083 & 0.0625 & 0.05 & 0.042 & 0.035 & 0.031 & 0.027 & 0.025 \\
\hline
\end{tabular}




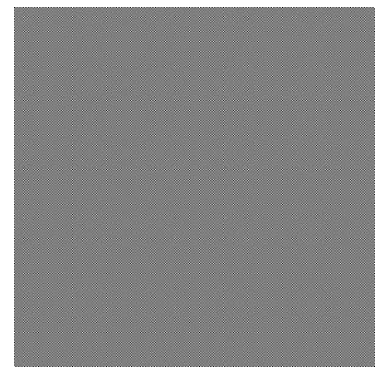

A. width: $4(512 \times 512)$

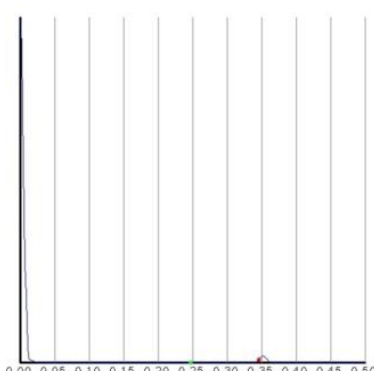

A-4. SIVV (4; location 0.35)

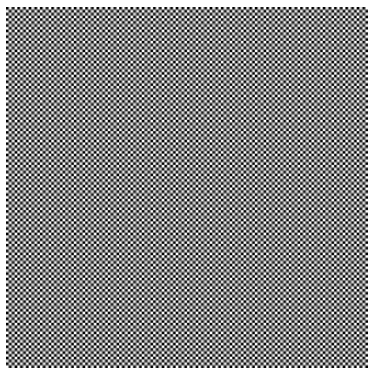

B. width: $8(512 \times 512)$

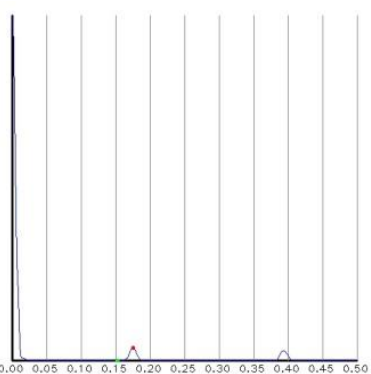

B-4. SIVV (8; location 0.175)

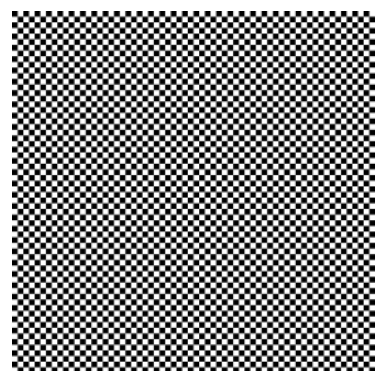

C. width: $16(512 \times 512)$



C-4. SIVV (16; location:0.085)

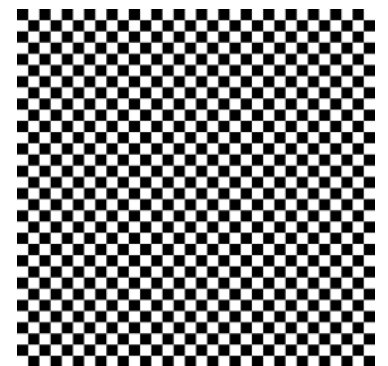

D. width: $32(512 \times 512)$

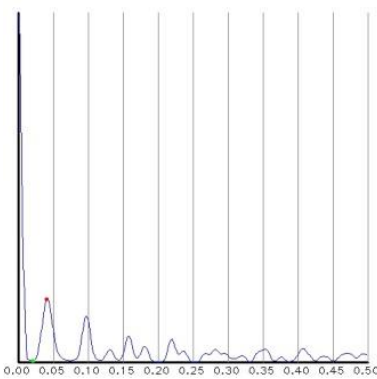

D-4. SIVV (32; Around 0.04)

Figure 27: SIVV on the images with square pattern. 